TAIWANESE JOURNAL OF MATHEMATICS

Vol. 14, No. 3B, pp. 1201-1248, June 2010

This paper is available online at http://www.tjm.nsysu.edu.tw/

\title{
GROWTH ORDERS OF CESÀRO AND ABEL MEANS OF FUNCTIONS IN BANACH SPACES
}

\author{
Jeng-Chung Chen, Ryotaro Sato and Sen-Yen Shaw*
}

\begin{abstract}
For continuous vector-valued functions, we discuss relations among exponential and polynomial growth orders of the $\gamma$-Cesaro mean $(\gamma \geq 0)$ and of the Abel mean. In general, the Abel mean has growth order not larger than those of Cesàro means, and a higher-order Cesàro mean has a smaller growth order than a lower-order Cesaro mean. But, for a positive function in a Banach lattice, the Abel mean and all $\gamma$-Cesaro means with $\gamma \geq 1$ (but not with $0 \leq \gamma<1$ ) have the same polynomial growth order. The possibility of non-equal growth orders for these means is illustrated by some examples of $C_{0}$-semigroups and cosine operator functions.
\end{abstract}

\section{INTRODUCTION}

Let $u \in C([0, \infty), X)$ be a continuous function with values in a Banach space $X$. For $\gamma \geq 0, t \geq 0$, the $\gamma$-th order Cesàro mean (or $(C, \gamma)$-mean) $\mathfrak{c}_{t}^{\gamma}$ of $u$ over $[0, t]$ is defined as $\mathfrak{c}_{0}^{\gamma}:=u(0)$ and, for $t>0$,

$$
\begin{aligned}
\mathfrak{c}_{t}^{\gamma}=\mathfrak{c}_{t}^{\gamma}(u): & = \begin{cases}u(t) & \text { if } \gamma=0, \\
\gamma t^{-\gamma} \int_{0}^{t}(t-s)^{\gamma-1} u(s) d s & \text { if } \gamma>0\end{cases} \\
& =\left(k_{\gamma+1}(t)\right)^{-1}\left(k_{\gamma} * u\right)(t),
\end{aligned}
$$

where $k_{0}:=\delta_{0}$, the Dirac measure at 0 , and $k_{\gamma}(t):=t^{\gamma-1} / \Gamma(\gamma)$ for $\gamma>0$. For $\lambda \in \mathbb{C}$ with $\operatorname{Re} \lambda>0$ the Abel mean $\mathfrak{a}_{\lambda}$ of $u$ is defined as

$$
\mathfrak{a}_{\lambda}=\mathfrak{a}_{\lambda}(u):=\lambda \int_{0}^{\infty} e^{-\lambda s} u(s) d s=\lambda \lim _{t \rightarrow \infty} \int_{0}^{t} e^{-\lambda s} u(s) d s
$$

Received May 13, 2009.

2000 Mathematics Subject Classification: 40E10, 40G05, 40G10, 46B99, 46B15, 47A35, 47A63.

Key words and phrases: Ces àro mean, Abel mean, Exponential growth order, Polynomial growth order, $C_{0}$-semigroup, Cosine operator function.

*Research partially supported by the National Science Council of Taiwan. 
if the limit exists. The abscissa of convergence $\sigma(u)$ of the Laplace integral $\widehat{u}(\lambda)=$ $\int_{0}^{\infty} e^{-\lambda s} u(s) d s:=\lim _{t \rightarrow \infty} \int_{0}^{t} e^{-\lambda s} u(s) d s$ is defined as

$$
\sigma(u):=\inf \left\{\operatorname{Re} \lambda: \lim _{t \rightarrow \infty} \int_{0}^{t} e^{-\lambda s} u(s) d s \text { exists }\right\} .
$$

Note that if $\int_{0}^{\infty} e^{-\lambda s} u(s) d s$ exists as a Bochner integral, then it agrees with the definition of Laplace integral by the dominated convergence theorem. $u$ is said to be Laplace transformable if $\sigma(u)<\infty$. The mapping $\widehat{u}: \lambda \mapsto \widehat{u}(\lambda)$ is called the Laplace transform of $u$. It is known that if $\lambda \in \mathbb{C}$ satisfies $\operatorname{Re} \lambda>\sigma(u)$, then the Laplace integral $\widehat{u}(\lambda)=\lim _{t \rightarrow \infty} \int_{0}^{t} e^{-\lambda s} u(s) d s$ exists, and the Laplace transform $\widehat{u}: \lambda \mapsto \widehat{u}(\lambda)$ is analytic on the domain $\{\lambda \in \mathbb{C}: \operatorname{Re} \lambda>\sigma(u)\}$ (cf. [1, Theorems 1.4.1 and 1.5.1]). We mainly consider $\mathfrak{a}_{\lambda}$ with $\lambda \in \mathbb{R}$ such that $\lambda>\max \{\sigma(u), 0\}$.

For fixed $u$ we use $\mathfrak{c}^{\gamma}$ and $\mathfrak{a}$. to denote the continuous functions $t \mapsto \mathfrak{c}_{t}^{\gamma}(u)$ and $\lambda \mapsto \mathfrak{a}_{\lambda}(u)$, respectively. When we consider a strongly continuous operator-valued function $t \mapsto T(t)(\in B(X))$ on $[0, \infty)$, such as a $C_{0}$-semigroup $(T(t))_{t \geq 0}$ of bounded linear operators on $X$, we set $u(t):=T(t) x$, where $x \in X$, and use the notations $C_{t}^{\gamma} x$ and $A_{\lambda} x$ instead of $\mathfrak{c}_{t}^{\gamma}(T(\cdot) x)$ and $\mathfrak{a}_{\lambda}(T(\cdot) x)$, respectively. Clearly, the mapping $C_{t}^{\gamma}: x \mapsto C_{t}^{\gamma} x$ becomes a bounded linear operator on $X$. If $A_{\lambda} x$ exists for all $x \in X$, then the mapping $A_{\lambda}: x \mapsto A_{\lambda} x$ is also a bounded linear operator on $X$ by the uniform boundedness principle.

One of the important issues of ergodic theory is concerned with convergence of the Cesaro mean and the Abel mean of operator-valued functions. It is wellknown that convergence of a function implies convergence of its Cesaro mean, and the latter implies convergence of the Abel mean (cf. [6, Theorem 18.2.1]), but not conversely (cf. [7, pp. 115-116]). This result seems natural if one notices the fact that the Abel mean is dominated by the Cesaro mean, and the latter is dominated by the function itself (cf. [8, Proposition 2.1]). Recently, some papers appeared discussing characterizations of bounded and polynomially bounded $C_{0}$-semigroups in terms of boundedness conditions on their Abel means (cf. [3, 4, 5, 9]). In [8] we have compared the growth orders of $C_{t}^{1}$ and $A_{\lambda}$ for a $C_{0}$-semigroup. In this paper we continue the investigation of $\mathfrak{c}_{t}^{\gamma}(u)$ for all $\gamma \geq 0$ and for a general vector-valued function $u$.

We need to introduce some more definitions. The function $u$ is said to be exponentially bounded if $\|u(t)\| \leq M e^{w t}$ for some $M \geq 1, w \in \mathbb{R}$ and all $t \geq 0$. In this case, we can define the exponential growth order (or bound):

$$
w_{0}(u):=\inf \left\{w \in \mathbb{R}:\|u(t)\|=O\left(e^{w t}\right)(t \rightarrow \infty)\right\}<\infty .
$$

Clearly, $\int_{0}^{\infty} e^{-\lambda t} u(t) d t$ exists as a Bochner integral for all $\lambda$ with $\operatorname{Re} \lambda>w_{0}(u)$. It follows that $\sigma(u) \leq w_{0}(u)$. If $w_{0}(u) \leq 0$, then $u$ is said to be sub-exponential. If $\sigma(u) \leq 0$, then one can define the growth order $\alpha_{0}(\mathfrak{a}$.) of $\mathfrak{a}$. at 0 as

$$
\alpha_{0}(\mathfrak{a} .):=\inf \left\{\alpha \in \mathbb{R}:\left\|\mathfrak{a}_{\lambda}\right\|=O\left(\lambda^{-\alpha}\right)(\lambda \downarrow 0)\right\} .
$$


One can also define the polynomial growth order of $u$ as

$$
\alpha_{0}(u):=\inf \left\{\alpha \in \mathbb{R}:\|u(t)\|=O\left(t^{\alpha}\right)(t \rightarrow \infty)\right\} .
$$

If $\alpha_{0}(u)<\infty$, then $u$ is said to be polynomially bounded.

It would be interesting to find the relations among $w_{0}(u)$ and $w_{0}\left(\mathfrak{c}^{\gamma}\right)(0<\gamma<$ $\infty)$, and the relations among $\alpha_{0}(u), \alpha_{0}\left(\mathfrak{c}^{\gamma}\right)(0<\gamma<\infty)$, and $\alpha_{0}(\mathfrak{a}$.$) . The aim of$ this paper is to investigate various growth phenomena of functions.

Section 2 is concerned with general relations among growth orders and the abscissa of convergence. In particular, both $\max \left\{w_{0}\left(\mathfrak{c}^{\gamma} \cdot\right), 0\right\}$ and $\max \left\{\sigma\left(\mathfrak{c}^{\gamma}\right), 0\right\}$ are non-increasing functions of $\gamma$ on $[0, \infty)$, and $\max \left\{w_{0}\left(\mathfrak{c}^{\gamma}\right), 0\right\}=\max \left\{\sigma\left(\mathfrak{c}^{\gamma-1}\right), 0\right\}$ for all $\gamma \geq 1$ (Theorems 2.2 and 2.3). Moreover, if $u \neq 0$ is a positive function in a Banach lattice, then we have (Remark under Theorem 2.6)

$$
w_{0}\left(\mathfrak{c}_{.}^{\beta}\right) \geq w_{0}\left(\mathfrak{c}^{1}\right)=w_{0}\left(\mathfrak{c}^{\gamma}\right)=\sigma\left(\mathfrak{c}^{\gamma-1}\right)=\max \{\sigma(u), 0\}
$$

for all $0<\beta<1$ and $\gamma>1$, where the first inequality may be strict, and it may happen that $w_{0}\left(\mathfrak{c}^{1}\right)=0>w_{0}\left(\mathfrak{c}^{0}\right)=w_{0}(u)>\sigma(u)$. Regarding the polynomial growth orders, we see that if $\sigma(u) \leq 0$ then the inequalities

$$
\alpha_{0}(\mathfrak{a} .) \leq \max \left\{\alpha_{0}\left(\mathfrak{c}^{\gamma^{\prime}}\right),-1-\gamma^{\prime}\right\} \leq \max \left\{\alpha_{0}\left(\mathfrak{c}^{\gamma}\right),-1-\gamma\right\}
$$

hold for all $0 \leq \gamma<\gamma^{\prime}<\infty$ (Corollary 2.8). In addition, if $u$ is a positive function in a Banach lattice then the equivalence

$$
\sup _{\lambda>0}\left\|\lambda^{\alpha} \mathfrak{a}_{\lambda}\right\|<\infty \Leftrightarrow \sup _{t>0}\left\|t^{-\alpha} \mathfrak{c}_{t}^{\gamma}\right\|<\infty
$$

holds for all $\gamma \geq 1$ whenever $\alpha>-2$ (Corollary 2.10).

Two questions come up naturally: (A) When do the inequalities in (5) become equalities and for what examples will some of the inequalities become strict? (B) Does the equivalence in (6) hold for $0 \leq \gamma<1$ ? To answer these questions, we turn to consider in Sections 3 and 4 growth orders of means of a $C_{0}$-semigroup $(T(t))_{t \geq 0}$ of bounded linear operators on a Banach space $X$. In Section 3 we see that for each integer $k \geq 1$ there exists a $C_{0}$-semigroup $(T(t))_{t \geq 0}$ such that $\left\|C_{t}^{n}\right\| \sim t^{k-n}$ $(t \rightarrow \infty)$ for $n=0,1, \ldots, k,\left\|C_{t}^{\gamma}\right\| \sim t^{-1}(t \rightarrow \infty)$ for all $\gamma \geq k+1$, $\left\|A_{\lambda}\right\| \sim \lambda(\lambda \downarrow 0), \sup _{t>0}\left\|C_{t}^{\gamma}\right\|<\infty$ for all $\gamma \geq k$, and $\sup _{t>0}\left\|C_{t}^{\gamma}\right\|=\infty$ for all $\gamma$ with $0 \leq \gamma<k$ (Theorems 3.4 and 3.5). Here, $a(t) \sim b(t)(t \rightarrow \infty)$ [resp. $(t \downarrow 0)]$ means that both the ratios $a(t) / b(t)$ and $b(t) / a(t)$ are bounded in some interval $(\delta, \infty)$ [resp. $(0, \delta)$ ]. In Section 4 we see that for any $0 \leq \gamma<1$ there exists a $C_{0}$-semigroup $(T(t))_{t \geq 0}$ of positive linear operators on an $L_{1}$-space such that $\sup _{t>0}\left\|C_{t}^{\gamma}\right\|=\infty$, but $\sup _{t>0}\left\|C_{t}^{\beta}\right\|<\infty$ for all $\beta>\gamma$ and, in particular, the Abel means of $(T(t))_{t \geq 0}$ are uniformly bounded (Theorem 4.2). 


\section{Estimates of Growth Orders}

In this section we compare growth orders of the Cesaro means $\mathfrak{c}_{t}^{\gamma}$, with $\gamma \geq 0$, and the Abel means $\mathfrak{a}_{\lambda}(\lambda>\max \{\sigma(u), 0\})$ of a function $u \in C([0, \infty), X)$. To do this we start with the following

Lemma 2.1. For all $\gamma \geq 0$, the Laplace transform of $k_{\gamma}$ satisfies $\widehat{k}_{\gamma}(\lambda)=$ $\lambda^{-\gamma}, \lambda>0$, and therefore $\widehat{k}_{r+s}=\widehat{k}_{r} \widehat{k}_{s}$ so that $k_{r} * k_{s}=k_{r+s}$ for all $r, s \geq 0$, where $k_{r} * k_{s}$ denotes the convolution of $k_{r}$ and $k_{s}$.

Proof. If $\gamma=0$, then $\widehat{k}_{0}(\lambda):=\int_{0}^{\infty} e^{-\lambda t} d \delta_{0}(t)=1=\lambda^{0}$ for all $\lambda>0$. Next, if $\gamma>0$, then

$$
\widehat{k}_{\gamma}(\lambda)=\int_{0}^{\infty} e^{-\lambda t} k_{\gamma}(t) d t=\frac{1}{\Gamma(\gamma)} \int_{0}^{\infty} e^{-\lambda t} t^{\gamma-1} d t=\lambda^{-\gamma}
$$

for all $\lambda>0$. Hence, for all $\lambda>0$ and $r, s \geq 0$, we have

$$
\widehat{k}_{r+s}(\lambda)=\lambda^{-r-s}=\lambda^{-r} \cdot \lambda^{-s}=\widehat{k}_{r}(\lambda) \widehat{k}_{s}(\lambda)=\left(\widehat{k_{r} * k_{s}}\right)(\lambda),
$$

so that, by the uniqueness of the Laplace transform, $k_{r} * k_{s}=k_{r+s}$.

We first state results on exponential growth orders and their relations to the abscissa of convergence.

Theorem 2.2. Let $u \in C([0, \infty), X)$ and $\gamma \geq 0$. Then the following hold.

(i) $\left\|\mathfrak{c}_{t}^{\gamma+\beta}\right\| \leq \sup _{0 \leq s \leq t}\left\|\mathfrak{c}_{s}^{\gamma}\right\|$ for all $\beta>0$ and $t>0$.

(ii) If $\left\|\mathfrak{c}_{t}^{\gamma}\right\| \leq M e^{w t}$ for some $M>0$ and $w \geq 0$ and all $t>0$, then $\left\|\mathfrak{c}_{t}^{\gamma+\beta}\right\| \leq$ $M e^{w t}$ for all $\beta>0$ and $t>0$. Thus $\max \left\{w_{0}\left(\mathfrak{c}^{\gamma+\beta}\right), 0\right\} \leq \max \left\{w_{0}\left(\mathfrak{c}^{\gamma}\right), 0\right\}$, that is, the function $\gamma \mapsto \max \left\{w_{0}\left(\mathfrak{c}^{\gamma}\right), 0\right\}$ is non-increasing on $[0, \infty)$.

Proof. (i) Using Lemma 2.1 and (1), we can write

$$
\begin{aligned}
\left\|\mathfrak{c}_{t}^{\gamma+\beta}\right\| & =\left(k_{\gamma+\beta+1}(t)\right)^{-1}\left\|\left(k_{\gamma+\beta} * u\right)(t)\right\| \\
& =\left(k_{\gamma+\beta+1}(t)\right)^{-1}\left\|\left(k_{\beta} * k_{\gamma} * u\right)(t)\right\| \\
& =\left(k_{\gamma+\beta+1}(t)\right)^{-1}\left\|\left(k_{\beta} *\left(k_{\gamma+1} \mathfrak{c}^{\gamma}\right)\right)(t)\right\| \\
& \leq\left(k_{\gamma+\beta+1}(t)\right)^{-1}\left(k_{\beta} * k_{\gamma+1}\right)(t) \sup _{0 \leq s \leq t}\left\|\mathfrak{c}_{s}^{\gamma}\right\| \\
& =\sup _{0 \leq s \leq t}\left\|\mathfrak{c}_{s}^{\gamma}\right\| .
\end{aligned}
$$


(ii) Note that $\left\|\mathfrak{c}_{t}^{\gamma}\right\|=O\left(e^{w t}\right)(t \rightarrow \infty)$ is equivalent to $\left\|\mathfrak{c}_{t}^{\gamma}\right\| \leq M e^{w t}$ for some $M>0$ and all $t>0$ because $u$ is assumed to be continuous on $[0, \infty)$. Hence the assertion follows immediately from (i).

Theorem 2.3. Let $u \in C([0, \infty), X)$. Then the following hold.

(i) For all $\gamma \geq 0$ we have

$$
\begin{aligned}
\max \left\{\sigma\left(\mathfrak{c}^{\gamma}\right), 0\right\} & =\max \left\{\sigma\left(k_{\gamma} * u\right), 0\right\} \\
& =\max \left\{w_{0}\left(k_{\gamma+1} * u\right), 0\right\}=\max \left\{w_{0}\left(\mathfrak{c}^{\gamma+1}\right), 0\right\} .
\end{aligned}
$$

Thus the function $\gamma \mapsto \max \left\{\sigma\left(\mathfrak{c}^{\gamma}\right), 0\right\}$ is non-increasing on $[0, \infty)$.

(ii) The identities

$$
\mathfrak{a}_{\lambda}=\lambda^{\gamma+1} \int_{0}^{\infty} e^{-\lambda t}\left(k_{\gamma} * u\right)(t) d t=\lambda^{\gamma+1} \int_{0}^{\infty} e^{-\lambda t} k_{\gamma+1}(t) \mathfrak{c}_{t}^{\gamma} d t
$$

hold for all $\gamma \geq 0$ and $\lambda \in \mathbb{C}$ with $\operatorname{Re} \lambda>\max \{\sigma(u), 0\}$.

Proof. (i) We first see that if $p$ is a measurable function on $(0, \infty)$ such that $w_{0}(p)=w_{0}(1 / p)=0$ then

$$
w_{0}(p u)=w_{0}(u)
$$

for all $u \in C([0, \infty), X)$. In fact, it follows from $w_{0}(p)=0$ that $w_{0}(p u) \leq$ $w_{0}(p)+w_{0}(u)=w_{0}(u)$. Similarly, $w_{0}(u) \leq w_{0}(p u)$ follows from $w_{0}(1 / p)=0$.

Next, we see that if $p$ is a differentiable function such that $w_{0}(p)=w_{0}\left(p^{\prime}\right)=0$ and if $\int_{0}^{1}\|p(s) u(s)\| d s<\infty$, then

$$
\sigma(p u) \leq \sigma(u)
$$

In fact, the assumption implies that $\int_{0}^{1} e^{-\lambda s} p(s) u(s) d s$ exists for any $\lambda$. Take any $\lambda>\sigma(u)$. Then $\widehat{u}\left(\lambda_{0}\right)$ exists at some $\lambda_{0} \in(\sigma(u), \lambda)$, so that the function $v(t):=\int_{0}^{t} e^{-\lambda_{0} s} u(s) d s$ is bounded on $[0, \infty)$. Using integration by parts, we have

$$
\begin{aligned}
\int_{1}^{t} e^{-\lambda s} p(s) u(s) d s= & \int_{1}^{t} e^{-\left(\lambda-\lambda_{0}\right) s} p(s) e^{-\lambda_{0} s} u(s) d s \\
= & e^{-\left(\lambda-\lambda_{0}\right) t} p(t) v(t)-e^{-\left(\lambda-\lambda_{0}\right)} p(1) v(1) \\
& +\left(\lambda-\lambda_{0}\right) \int_{1}^{t} e^{-\left(\lambda-\lambda_{0}\right) s} p(s) v(s) d s \\
& -\int_{1}^{t} e^{-\left(\lambda-\lambda_{0}\right) s} p^{\prime}(s) v(s) d s
\end{aligned}
$$


for all $t \geq 1$. Since $v$ is bounded and $w_{0}(p)=w_{0}\left(p^{\prime}\right)=0$, it follows that $\int_{1}^{\infty} e^{-\lambda s} p(s) u(s) d s$ exists and

$$
\begin{aligned}
\int_{1}^{\infty} e^{-\lambda s} p(s) u(s) d s= & -e^{-\left(\lambda-\lambda_{0}\right)} p(1) v(1)+\left(\lambda-\lambda_{0}\right) \int_{1}^{\infty} e^{-\left(\lambda-\lambda_{0}\right) s} p(s) v(s) d s \\
& -\int_{1}^{\infty} e^{-\left(\lambda-\lambda_{0}\right) s} p^{\prime}(s) v(s) d s
\end{aligned}
$$

Hence $\widehat{(p u)}(\lambda)$ exists. This shows $\sigma(p u) \leq \sigma(u)$.

Since $w_{0}\left(k_{\beta}\right)=w_{0}\left(1 / k_{\beta}\right)=w_{0}\left(k_{\beta}^{\prime}\right)=w_{0}\left(\left(1 / k_{\beta}\right)^{\prime}\right)=0, \int_{0}^{1}\left\|k_{\beta}(s) u(s)\right\| d s<$ $\infty$ and $\int_{0}^{1}\left\|\left(k_{\beta-1} * u\right)(s) / k_{\beta}(s)\right\| d s=\int_{0}^{1}\left\|\mathfrak{c}_{s}^{\beta-1}\right\| d s<\infty$ for all $\beta \geq 1$ and $u \in C([0, \infty), X)$, applications of (2.3) (with $p=k_{\gamma+1}$ ) and (2.4) (with $p=k_{\gamma+1}$ and $\left.p=1 / k_{\gamma+1}\right)$ yield that

$$
\begin{gathered}
w_{0}\left(\mathfrak{c}^{\gamma}\right)=w_{0}\left(k_{\gamma+1} \mathfrak{c}^{\gamma}\right)=w_{0}\left(k_{\gamma} * u\right) \\
\sigma\left(\mathfrak{c}^{\gamma}\right)=\sigma\left(\left(k_{\gamma} * u\right) / k_{\gamma+1}\right) \leq \sigma\left(k_{\gamma} * u\right)=\sigma\left(k_{\gamma+1} \mathfrak{c}^{\gamma}\right) \leq \sigma\left(\mathfrak{c}^{\gamma}\right)
\end{gathered}
$$

for all $\gamma \geq 0$.

It is known (cf. [1, Theorem 1.4.3]) that $u$ is Laplace transformable if and only if $1 * u$ is exponentially bounded (i.e. $\sigma(u)<\infty$ if and only if $w_{0}(1 * u)<\infty$ ), and $\sigma(u) \leq \lambda \Leftrightarrow w_{0}(1 * u) \leq \lambda$ (if $\lambda \geq 0$ ), that is, $\max \{\sigma(u), 0\}=\max \left\{w_{0}(1 * u), 0\right\}$. By this, together with (2.5) and (2.6), we deduce (2.1). Since $\max \left\{w_{0}\left(\mathfrak{c}^{\gamma+1}\right), 0\right\}$ is known to be non-increasing on $[0, \infty)$ (by Theorem 2.2(ii)), $\max \left\{\sigma\left(\mathfrak{c}^{\gamma}\right), 0\right\}$ is also non-increasing on $[0, \infty)$.

(ii) Since the case $\gamma=0$ is trivial, we consider the case $\gamma>0$. Let $\lambda \in \mathbb{C}$ be such that $\operatorname{Re} \lambda>\max \{\sigma(u), 0\}$. Then $\operatorname{Re} \lambda>\sigma\left(k_{\gamma} * u\right)$, and so

$$
\begin{aligned}
\int_{0}^{\infty} e^{-\lambda t}\left(k_{\gamma} * u\right)(t) d t & =\lambda \int_{0}^{\infty} e^{-\lambda t}\left(1 *\left(k_{\gamma} * u\right)\right)(t) d t \\
& =\lambda \int_{0}^{\infty} e^{-\lambda t}\left(k_{\gamma+1} * u\right)(t) d t
\end{aligned}
$$

(cf. the proof of Theorem 1.4.3 of [1]). Since $\operatorname{Re} \lambda>\max \{\sigma(u), 0\} \geq w_{0}(1 * u)$ implies

$$
\int_{0}^{\infty}\left|e^{-\lambda t}\right| \cdot\|(1 * u)(t)\| d t<\infty
$$

we then apply Fubini's theorem to obtain that 


$$
\begin{aligned}
\lambda \int_{0}^{\infty} e^{-\lambda t}\left(k_{\gamma+1} * u\right)(t) d t & =\lambda \int_{0}^{\infty} e^{-\lambda t}\left(k_{\gamma} *(1 * u)\right)(t) d t \\
& =\lambda \int_{0}^{\infty} \int_{0}^{t} e^{-\lambda(t-s)} k_{\gamma}(t-s) e^{-\lambda s}(1 * u)(s) d s d t \\
& =\lambda \int_{0}^{\infty} e^{-\lambda t} k_{\gamma}(t) d t \cdot \int_{0}^{\infty} e^{-\lambda s}(1 * u)(s) d s \\
& =\lambda \cdot \lambda^{-\gamma} \cdot \lambda^{-2} \mathfrak{a}_{\lambda}=\lambda^{-(\gamma+1)} \mathfrak{a}_{\lambda} .
\end{aligned}
$$

Hence

$$
\int_{0}^{\infty} e^{-\lambda t}\left(k_{\gamma} * u\right)(t) d t=\lambda^{-(\gamma+1)} \mathfrak{a}_{\lambda},
$$

and this completes the proof.

Remark. There exists a $u$ such that the function $\max \left\{\sigma\left(\mathfrak{c}^{\gamma}\right), 0\right\}$ is strictly decreasing in the sense that $\max \left\{\sigma_{0}\left(\mathfrak{c}^{\gamma}\right), 0\right\}>\max \left\{\sigma_{0}\left(\boldsymbol{c}^{\gamma^{\prime}}\right), 0\right\}$ for some $\gamma^{\prime}>\gamma \geq$ 0 . For example, if $u(t)=e^{t} \sin e^{t}+e^{2 t} \cos e^{t}$, then

$$
(1 * u)(t)=e^{t} \sin e^{t}-\sin 1, \quad\left(k_{2} * u\right)(t)=-\cos e^{t}-t \sin 1+\cos 1 .
$$

Thus $w_{0}(u)=2, w_{0}\left(\mathfrak{c}^{1}\right)=w_{0}(1 * u)=1=\sigma(u)=\sigma\left(\mathfrak{c}^{0}\right)$, and

$$
w_{0}\left(\mathfrak{c}^{2}\right)=w_{0}\left(k_{2} * u\right)=0=\sigma(1 * u)=\sigma\left(\mathfrak{c}^{1}\right), \sup _{t>0}\left|\mathfrak{c}_{t}^{2}\right|<\infty, \sup _{t>0} t\left|\mathfrak{c}_{t}^{2}\right|<\infty .
$$

It follows that $\sigma\left(\mathfrak{c}^{0}\right)=1>\sigma\left(\mathfrak{c}^{1}\right)=0$.

Theorem 2.4. Let $u \in C([0, \infty), X)$. Then the following hold.

(i) If $0 \leq \sigma(u)<w<\infty$, then $\sup _{\lambda>w}\left\|\mathfrak{a}_{\lambda}\right\|<\infty$.

(ii) If $\sigma(u)<0$, then $\sup _{\lambda>0}\left\|\lambda^{-1} \mathfrak{a}_{\lambda}\right\|<\infty$ and $\sup _{\lambda>0}\left\|\mathfrak{a}_{\lambda}\right\|<\infty$.

Proof. (i) Choose $\delta \in \mathbb{R}$ such that $\sigma(u)<\delta<w$. Then $\delta>\sigma(u)=$ $\max \{\sigma(u), 0\}=\max \left\{w_{0}\left(k_{1} * u\right), 0\right\}$, and so there exists $M_{\delta}>0$ such that $\|\left(k_{1} *\right.$ $u)(t) \| \leq M_{\delta} e^{\delta t}$ for all $t \geq 0$. Put $u_{1}(t)=\chi_{[1, \infty)}(t) u(t), F_{1}=\int_{0}^{1} u(s) d s$, and $M(1)=\max _{0 \leq t \leq 1}\|u(t)\|$. Then

$$
\left(k_{1} * u_{1}\right)(t)= \begin{cases}0 & \text { if } 0 \leq t \leq 1, \\ \left(k_{1} * u\right)(t)-F_{1} & \text { if } t>1\end{cases}
$$

Thus, for all $\lambda>w$,

$$
\mathfrak{a}_{\lambda}=\lambda \int_{0}^{\infty} e^{-\lambda t} u(t) d t=\lambda\left(\int_{0}^{1}+\int_{1}^{\infty}\right) e^{-\lambda t} u(t) d t=I+I I,
$$


where

$$
\|I\| \leq \lambda \int_{0}^{1} e^{-\lambda t} M(1) d t \leq M(1)
$$

and by Theorem 2.3(ii)

$$
\begin{aligned}
I I & =\lambda \int_{0}^{\infty} e^{-\lambda t} u_{1}(t) d t=\lambda^{2} \int_{0}^{\infty} e^{-\lambda t}\left(k_{1} * u_{1}\right)(t) d t \\
& =\lambda^{2} \int_{1}^{\infty} e^{-\lambda t}\left(\left(k_{1} * u\right)(t)-F_{1}\right) d t
\end{aligned}
$$

whence

$$
\begin{aligned}
\|I I\| & \leq \lambda^{2} \int_{1}^{\infty} e^{-(\lambda-\delta) t} M_{\delta} d t+\left\|F_{1}\right\| \lambda^{2} \cdot \frac{e^{-\lambda}}{\lambda} \\
& \leq M_{\delta} \frac{\lambda^{2}}{\lambda-\delta} e^{-(\lambda-\delta)}+\left\|F_{1}\right\| \lambda e^{-\lambda} \rightarrow 0 \quad \text { as } \lambda \rightarrow \infty .
\end{aligned}
$$

Therefore, $\lim \sup _{\lambda \rightarrow \infty}\left\|\mathfrak{a}_{\lambda}\right\|<\infty$. On the other hand, since

$$
\begin{aligned}
\left\|\mathfrak{a}_{\lambda}\right\| & =\lambda^{2}\left\|\int_{0}^{\infty} e^{-\lambda t}\left(k_{1} * u\right)(t) d t\right\| \\
& \leq M_{\delta} \lambda^{2} \int_{0}^{\infty} e^{-(\lambda-\delta) t} d t=M_{\delta} \lambda^{2}(\lambda-\delta)^{-1}
\end{aligned}
$$

it follows that $\lim \sup _{\lambda \downarrow w}\left\|\mathfrak{a}_{\lambda}\right\| \leq M_{\delta} w^{2}(w-\delta)^{-1}$. Hence, $\sup _{\lambda>w}\left\|\mathfrak{a}_{\lambda}\right\|<\infty$.

(ii) If $\sigma(u)<0$, then, by Theorem 2.3(ii),

$$
\mathfrak{a}_{\lambda}=\lambda \int_{0}^{\infty} e^{-\lambda t} u(t) d t=\lambda^{2} \int_{0}^{\infty} e^{-\lambda t}(1 * u)(t) d t
$$

for all $\lambda>0$. Since the hypothesis $\sigma(u)<0$ implies that $\lim _{t \rightarrow \infty}(1 * u)(t)=$ $\int_{0}^{\infty} u(s) d s$ exists, it follows that $M:=\sup _{t>0}\|(1 * u)(t)\|<\infty$. Hence

$$
\left\|\mathfrak{a}_{\lambda}\right\| \leq \lambda^{2} \int_{0}^{\infty} e^{-\lambda t} M d t=M \lambda
$$

for all $\lambda>0$. By this and the proof of (i) we also have $\sup _{\lambda>0}\left\|\mathfrak{a}_{\lambda}\right\|<\infty$.

The proof is complete.

Remarks. (1) The hypothesis $0 \leq \sigma(u)<w<\infty$ cannot be sharpened as $0 \leq \sigma(u)=w<\infty$ in Theorem 2.4(i). To see this, let $\lambda_{0} \geq 0$ and define $u(s):=e^{\lambda_{0} s} s$ for $s \geq 0$. Then $\sigma(u)=\lambda_{0}$ and, for all $\lambda>\lambda_{0}$, we have

$$
\mathfrak{a}_{\lambda}=\lambda \int_{0}^{\infty} e^{-\lambda s} u(s) d s=\lambda \int_{0}^{\infty} e^{-\left(\lambda-\lambda_{0}\right) s} s d s=\frac{\lambda}{\left(\lambda-\lambda_{0}\right)^{2}} .
$$


Since $\lim _{\lambda \downarrow \lambda_{0}} \lambda\left(\lambda-\lambda_{0}\right)^{-2}=\infty$, it follows that $\sup _{\lambda>\lambda_{0}}\left\|\mathfrak{a}_{\lambda}\right\|=\infty$.

(2) As for Theorem 2.4(ii) we note that $\alpha_{0}(\mathfrak{a}$. $)<-1$ cannot be expected in general. To see this, let $u(t):=\max \{2-t, 0\}$ for $t \geq 0$. Then $u \in C([0, \infty), \mathbb{R})$ and $w_{0}(u)=\alpha_{0}(u)=-\infty$. Since $u(t) \geq 1$ on $[0,1]$, it follows that $\mathfrak{a}_{\lambda} \geq$ $\lambda \int_{0}^{1} e^{-\lambda t} d t$ for all $\lambda>0$. Since $\lim _{\lambda \downarrow 0} \int_{0}^{1} e^{-\lambda t} d t=1$, it follows from Theorem 2.4(ii) that $\alpha_{0}$ (a.) $=-1$. (See also Corollary 2.10(ii).)

We will see that if $u \in C([0, \infty), X)$ is a positive function in a Banach lattice $X$, then the function $\gamma \mapsto \max \left\{\sigma\left(\mathfrak{c}^{\gamma}\right), 0\right\}$ is constant on $[0, \infty)$ (Theorem 2.6). To prove this we use the following lemma, which is stated in more general form than needed here.

Lemma 2.5. Let $X$ be a Banach lattice, and $u:(0, \infty) \rightarrow X$ be a positive $X$-valued strongly measurable function on $(0, \infty)$ such that $\int_{a}^{b}\|u(t)\| d t<\infty$ for all $0<a<b<\infty$ and $\int_{0}^{1} u(t) d t:=\lim _{\epsilon \downarrow 0} \int_{\epsilon}^{1} u(t) d t$ exists. Let $\lambda>0, \gamma>0$ and $x \in X$. Then

$$
\int_{0}^{\infty} e^{-\lambda t} u(t) d t\left(:=\lim _{b \rightarrow \infty} \int_{0}^{b} e^{-\lambda t} u(t) d t\right)=x
$$

if and only if

$$
\lambda^{\gamma} \int_{0}^{\infty} e^{-\lambda t}\left(k_{\gamma} * u\right)(t) d t=x .
$$

Hence $\max \{\sigma(u), 0\}=\max \left\{\sigma\left(k_{\gamma} * u\right), 0\right\}$.

Proof. It is immediate that $\left(k_{\gamma} * u\right)(t)$ exists for almost all $t>0$ and the mapping $t \mapsto\left(k_{\gamma} * u\right)(t)$ becomes a positive $X$-valued strongly measurable function on $(0, \infty)$. In order to prove the lemma it is necessary to show that $\int_{0}^{1}\left(k_{\gamma} * u\right)(t) d t=$ $\lim _{\epsilon \downarrow 0} \int_{\epsilon}^{1}\left(k_{\gamma} * u\right)(t) d t$ exists. To do this, let $0<\delta<\epsilon$. Then we have

$$
\begin{aligned}
\int_{\delta}^{\epsilon} & \left(k_{\gamma} * u\right)(t) d t \\
& =\int_{\delta}^{\epsilon}\left\{\int_{0}^{t} k_{\gamma}(t-s) u(s) d s\right\} d t \\
& =\lim _{\eta \downarrow 0} \int_{\delta}^{\epsilon}\left\{\int_{\eta}^{t} k_{\gamma}(t-s) u(s) d s\right\} d t \\
& =\lim _{\eta \downarrow 0} \int_{\eta}^{\delta}\left\{\int_{\delta}^{\epsilon} k_{\gamma}(t-s) d t\right\} u(s) d s+\int_{\delta}^{\epsilon}\left\{\int_{s}^{\epsilon} k_{\gamma}(t-s) d t\right\} u(s) d s \\
& =\int_{0}^{\delta}\left\{\int_{\delta}^{\epsilon} k_{\gamma}(t-s) d t\right\} u(s) d s+\int_{\delta}^{\epsilon}\left\{\int_{s}^{\epsilon} k_{\gamma}(t-s) d t\right\} u(s) d s .
\end{aligned}
$$


For any $\eta>0$ we can choose $\epsilon^{\sim}>0$ so that

$$
\int_{0}^{\epsilon^{\sim}} k_{\gamma}(s) d s<\eta \quad \text { and } \quad\left\|\int_{0}^{\epsilon^{\sim}} u(s) d s\right\|<\eta
$$

Then, for $0<\delta<\epsilon<\epsilon^{\sim}$ we have

$$
\begin{aligned}
\left\|\int_{\delta}^{\epsilon}\left(k_{\gamma} * u\right)(t) d t\right\| \leq & \left\|\int_{0}^{\delta}\left\{\int_{\delta}^{\epsilon} k_{\gamma}(t-s) d t\right\} u(s) d s\right\| \\
& +\left\|\int_{\delta}^{\epsilon}\left\{\int_{s}^{\epsilon} k_{\gamma}(t-s) d t\right\} u(s) d s\right\| \\
& <\eta\left\|\int_{0}^{\delta} u(s) d s\right\|+\eta\left\|\int_{\delta}^{\epsilon} u(s) d s\right\|<2 \eta^{2} .
\end{aligned}
$$

This implies that $\left\|\int_{\delta}^{\epsilon}\left(k_{\gamma} * u\right)(t) d t\right\| \rightarrow 0$ as $\epsilon \downarrow 0$ with $0<\delta<\epsilon$. Hence $\int_{0}^{1}\left(k_{\gamma} * u\right)(t) d t=\lim _{\epsilon \downarrow 0} \int_{\epsilon}^{1}\left(k_{\gamma} * u\right)(s) d s$ exists.

Suppose $\int_{0}^{\infty} e^{-\lambda t} u(t) d t=x$. We first prove that

$$
\text { weak- } \lim _{b \rightarrow \infty} \lambda^{\gamma} \int_{0}^{b} e^{-\lambda t}\left(k_{\gamma} * u\right)(t) d t=x \text {. }
$$

Let $x^{*}$ be any element of $X^{*}$, where $X^{*}$ denotes the dual space of $X$. It suffices to show that

$$
\lambda^{\gamma} \int_{0}^{\infty} e^{-\lambda t}\left\langle\left(k_{\gamma} * u\right)(t), x^{*}\right\rangle d t=\lim _{b \rightarrow \infty} \lambda^{\gamma} \int_{0}^{b} e^{-\lambda t}\left\langle\left(k_{\gamma} * u\right)(t), x^{*}\right\rangle d t=\left\langle x, x^{*}\right\rangle .
$$

Here, if necessary, we may consider the real part of $x^{*}$ without loss of generality. Thus we may assume that $x^{*}$ is a real-linear functional on $X$. Then $x^{*}$ can be written as $x^{*}=x_{1}^{*}-x_{2}^{*}$, where $x_{1}^{*}$ and $x_{2}^{*}$ are positive real-linear functionals on $X$. Therefore, from the first, we may assume that $x^{*}$ is a positive real-linear functional on $X$. Then, since $\left\langle u(t), x^{*}\right\rangle \geq 0$ for all $t \geq 0$ and $\int_{0}^{\infty} e^{-\lambda t}\left\langle u(t), x^{*}\right\rangle d t=\left\langle x, x^{*}\right\rangle$, Fubini's theorem implies that

$$
\begin{aligned}
& \lambda^{\gamma} \int_{0}^{\infty} e^{-\lambda t}\left\langle\left(k_{\gamma} * u\right)(t), x^{*}\right\rangle d t \\
& \quad=\lambda^{\gamma} \int_{0}^{\infty} \int_{0}^{t} e^{-\lambda(t-s)} k_{\gamma}(t-s) e^{-\lambda s}\left\langle u(s), x^{*}\right\rangle d s d t \\
& =\lambda^{\gamma} \int_{0}^{\infty} e^{-\lambda t} k_{\gamma}(t) d t \cdot \int_{0}^{\infty} e^{-\lambda s}\left\langle u(s), x^{*}\right\rangle d s \\
& =\lambda^{\gamma} \lambda^{-\gamma} \cdot \int_{0}^{\infty} e^{-\lambda s}\left\langle u(s), x^{*}\right\rangle d s=\left\langle x, x^{*}\right\rangle .
\end{aligned}
$$


Hence $\lambda^{\gamma} \int_{0}^{b} e^{-\lambda t}\left(k_{\gamma} * u\right)(t) d t \leq x$ for all $b>0$, and

$$
\text { weak- } \lim _{b \rightarrow \infty} \lambda^{\gamma} \int_{0}^{b} e^{-\lambda t}\left(k_{\gamma} * u\right)(t) d t=x .
$$

By this together with the fact that the weak closure and the strong closure of a convex subset of $X$ are identical, given $\epsilon>0$ and $G>0$, we can choose $b_{j}>G$ and $c_{j}>0(j=1,2, \ldots, n)$ such that $\sum_{j=1}^{n} c_{j}=1$ and

$$
\left\|x-\lambda^{\gamma} \sum_{j=1}^{n} c_{j} \int_{0}^{b_{j}} e^{-\lambda t}\left(k_{\gamma} * u\right)(t) d t\right\|<\epsilon .
$$

Then, for all $b>\max \left\{b_{1}, b_{2}, \ldots, b_{n}\right\}$, we have

$$
\lambda^{\gamma} \sum_{j=1}^{n} c_{j} \int_{0}^{b_{j}} e^{-\lambda t}\left(k_{\gamma} * u\right)(t) d t \leq \lambda^{\gamma} \int_{0}^{b} e^{-\lambda t}\left(k_{\gamma} * u\right)(t) d t \leq x
$$

and thus

$$
\left\|x-\lambda^{\gamma} \int_{0}^{b} e^{-\lambda t}\left(k_{\gamma} * u\right)(t) d t\right\| \leq\left\|x-\lambda^{\gamma} \sum_{j=1}^{n} c_{j} \int_{0}^{b_{j}} e^{-\lambda t}\left(k_{\gamma} * u\right)(t) d t\right\|<\epsilon
$$

which proves that $\lambda^{\gamma} \int_{0}^{\infty} e^{-\lambda t}\left(k_{\gamma} * u\right)(t) d t=x$.

The converse implication is also proved by the same argument, and hence we omit the details.

Theorem 2.6. Let $X$ be a Banach lattice and $0 \neq u \in C([0, \infty), X)$ be a positive $X$-valued function. Then $\sigma\left(\mathfrak{c}^{\gamma}\right)=\sigma(u)$ for all $\gamma>0$ if $\sigma(u)>0$, and $\sigma\left(\mathfrak{c}^{\gamma}\right)=0$ for all $\gamma>0$ if $\sigma(u) \leq 0$.

Proof. Suppose $\sigma(u)>0$. Then it follows from (2.6) and Lemma 2.5 that $\sigma\left(\mathfrak{c}^{\gamma}\right)=\sigma\left(k_{\gamma} * u\right)=\sigma(u)>0$ for all $\gamma>0$. Next, suppose $\sigma(u) \leq 0$ and $\gamma>0$. Then, since $u \neq 0$ by hypothesis, it follows that $\int_{0}^{\infty} e^{-\lambda t} u(t) d t \geq$ $\int_{0}^{\infty} e^{-\beta t} u(t) d t>0$ for all $\beta>\lambda>0$. Therefore Lemma 2.5 yields

$$
\lim _{\lambda \downarrow 0}\left\|\int_{0}^{\infty} e^{-\lambda t}\left(k_{\gamma} * u\right)(t) d t\right\|=\lim _{\lambda \downarrow 0} \lambda^{-\gamma}\left\|\int_{0}^{\infty} e^{-\lambda t} u(t) d t\right\|=\infty
$$

which proves that $\sigma\left(\mathfrak{c}^{\gamma}\right)=\sigma\left(k_{\gamma} * u\right)=0$. 
Remark. Let $u \neq 0$ be a positive function. Then, by Theorems 2.6 and 2.3, $\max \left\{w_{0}\left(\mathfrak{c}^{1}\right), 0\right\}=\max \{\sigma(u), 0\}=\sigma\left(\mathfrak{c}^{\gamma-1}\right)=\max \left\{w_{0}\left(\mathfrak{c}^{\gamma}\right), 0\right\}$ for all $\gamma>1$. Further, there exists $K>0$ such that $\int_{0}^{K} u(s) d s>0$. Thus, if $0<\gamma \leq 1$, then

$$
\mathfrak{c}_{t}^{\gamma}=\frac{\gamma}{t^{\gamma}} \int_{0}^{t}(t-s)^{\gamma-1} u(s) d s \geq \frac{\gamma}{t^{\gamma}} \cdot t^{\gamma-1} \int_{0}^{t} u(s) d s \geq \frac{\gamma}{t} \int_{0}^{K} u(s) d s \neq 0
$$

for all $t>K$, which implies $w_{0}\left(\mathfrak{c}^{\gamma}\right) \geq 0$. By a similar calculation, if $\gamma>1$ then $w_{0}\left(\mathfrak{c}^{\gamma}\right) \geq 0$. Thus, by Theorem 2.2, the function $\gamma \mapsto w_{0}\left(\mathfrak{c}^{\gamma}\right)$ is non-increasing on $(0, \infty)$, and $w_{0}\left(\mathfrak{c}^{\gamma}\right)=\max \{\sigma(u), 0\}$ for all $\gamma \geq 1$. (It may happen that $w_{0}\left(\mathfrak{c}^{1}\right)=$ $0>w_{0}\left(\mathfrak{c}^{0}\right)=w_{0}(u)>\sigma(u)$ for some positive $u \neq 0$.) Here we note that, for any given $0<\gamma \leq 1$, there exists a positive $u \neq 0$ such that $\sigma(u)=1-1 / \gamma \leq 0$, and

$$
w_{0}\left(\mathfrak{c}^{\beta}\right)= \begin{cases}1-\beta / \gamma & \text { if } 0 \leq \beta \leq \gamma \\ 0 & \text { if } \beta \geq \gamma .\end{cases}
$$

We give an example showing this.

Example 1. Let $N: \mathbb{N} \rightarrow \mathbb{N}$ be a strictly increasing function such that

$$
\lim _{n \rightarrow \infty} \frac{n}{N(n)}=0, \quad \text { and } \quad \lim _{n \rightarrow \infty} \frac{N(n+1)}{N(n)}=1
$$

(e.g. $N(n)=n^{2}$ satisfies (2.8)). For $n \in \mathbb{N}$, define $\delta_{n}$ with $0<\delta_{n}<1$ by

$$
\int_{N(n)-\delta_{n}}^{N(n)}(N(n)-s)^{\gamma-1} e^{s} d s=1 / 2^{n} .
$$

A simple calculation yields that

$$
\delta_{n} \sim \exp \{-(N(n)+n \ln 2) / \gamma\} \quad(n \geq 1),
$$

where $a(n) \sim b(n)(n \geq 1)$ means again that both the ratios $a(n) / b(n)$ and $b(n) / a(n)$ are bounded on $\mathbb{N}$.

Define

$$
A:=\bigcup_{n=1}^{\infty}\left[N(n)-\delta_{n}, N(n)\right), \quad \text { and } \quad u(s):=\chi_{A}(s) e^{s} \quad(s \geq 0) .
$$

Thus $u$ is a nonnegative function on $[0, \infty)$. Although $u$ is not a continuous function, $\mathfrak{c}_{t}^{\beta}=\mathfrak{c}_{t}^{\beta}(u)$ can be defined as in (1) for all $\beta \geq 0$ and $t \geq 0$. First we prove that $u$ satisfies (2.7). The following is a sketch of the proof. 
Since $s^{\gamma-1}$ is a non-increasing function on $[0, \infty)$, it follows from (2.9) that for $N(n)<t<N(n+1)$ we have

$$
\mathfrak{c}_{t}^{\gamma} \leq \frac{\gamma}{t^{\gamma}} \sum_{k=1}^{n+1} \int_{N(k)-\delta_{k}}^{N(k)}(N(k)-s)^{\gamma-1} e^{s} d s=\frac{\gamma}{t^{\gamma}} \sum_{k=1}^{n+1} \frac{1}{2^{k}} \leq \frac{\gamma}{t^{\gamma}},
$$

which tends to 0 as $t \rightarrow \infty$. Hence $w_{0}\left(\mathfrak{c}^{\gamma}\right)=0$, and so $w_{0}\left(\mathfrak{c}^{\beta}\right)=0$ for all $\beta \geq \gamma$. On the other hand, $w_{0}\left(\mathbf{c}^{0}\right)=w_{0}(u)=1$ is obvious from the definition of $u$.

Suppose $0<\beta<\gamma$. For $N(n)<t<N(n+1)$ we have

$$
\mathfrak{c}_{t}^{\beta} \leq \frac{\beta}{N(n)^{\beta}} \sum_{k=1}^{n+1} \int_{N(k)-\delta_{k}}^{N(k)}(N(k)-s)^{\beta-1} e^{s} d s,
$$

where by (2.10)

$$
\begin{aligned}
& \int_{N(k)-\delta_{k}}^{N(k)}(N(k)-s)^{\beta-1} e^{s} d s \sim e^{N(k)} \delta_{k}^{\beta} \\
& \quad \sim \exp \left\{N(k)\left(\left(1-\frac{\beta}{\gamma}\right)-\frac{\beta}{\gamma} \cdot \frac{k}{N(k)} \ln 2\right)\right\} \quad(k \geq 1)
\end{aligned}
$$

and by (2.8)

$$
\lim _{k \rightarrow \infty}\left(\left(1-\frac{\beta}{\gamma}\right)-\frac{\beta}{\gamma} \cdot \frac{k}{N(k)} \ln 2\right)=1-\frac{\beta}{\gamma}>0 .
$$

Thus, if $D>1-\beta / \gamma$ is fixed, then for all sufficiently large $k$

$$
e^{N(k)} \delta_{k}^{\beta} \leq \exp (N(k) D)
$$

Therefore

$$
\begin{aligned}
& \frac{\beta}{N(n)^{\beta}} \sum_{k=1}^{n+1} \int_{N(k)-\delta_{k}}^{N(k)}(N(k)-s)^{\beta-1} e^{s} d s \\
= & O(1) \cdot \frac{\beta}{N(n)^{\beta}} \sum_{k=1}^{N(n+1)} e^{k D} \\
\leq & O(1) \cdot \frac{\beta}{N(n)^{\beta}} \cdot \frac{1}{D} \exp \{(N(n+1)+1) D\} \\
= & o(1) \cdot \exp \left\{t D \cdot \frac{N(n+1)+1}{t}\right\} .
\end{aligned}
$$

Since

$$
\lim _{t \rightarrow \infty} \frac{N(n+1)+1}{t}=\lim _{n \rightarrow \infty} \frac{N(n+1)+1}{N(n)}=1 \quad(\text { by }(2.8)),
$$


it follows from (2.11) that $w_{0}\left(\mathfrak{c}^{\beta}\right) \leq D$ for all $D>1-\beta / \gamma$ so that

$$
w_{0}\left(\mathfrak{c}^{\beta}\right) \leq 1-\beta / \gamma
$$

Similarly the reverse inequality follows, and hence (2.7) follows for $u$. By (2.10) we also have $\int_{0}^{\infty} e^{-\eta s} u(s) d s=\sum_{n=1}^{\infty} \int_{N(n)-\delta_{n}}^{N(n)} e^{(1-\eta) s} d s<\infty$ if $(1-\eta)-1 / \gamma<0$, and $\int_{0}^{\infty} e^{-\eta s} u(s) d s=\infty$ if $(1-\eta)-1 / \gamma>0$. Thus $\sigma(u)=1-1 / \gamma$. By using this, it is now easy to find a continous $u$ on $[0, \infty)$ satisfying $\sigma(u)=1-1 / \gamma$ and (2.7). We may omit the details.

Next we turn to polynomial growth orders.

Theorem 2.7. Let $u \in C([0, \infty), X)$ and $\gamma \geq 0$. Then the following hold.

(i) If there exist $M>0$ and $\alpha>-1-\gamma$ such that $\left\|\mathfrak{c}_{t}^{\gamma}\right\| \leq M t^{\alpha}$ for all $t>0$, then

$$
\left\|\mathfrak{c}_{t}^{\gamma+\beta}\right\| \leq M \frac{\Gamma(\gamma+\alpha+1)}{\Gamma(\gamma+1)} \frac{\Gamma(\gamma+\beta+1)}{\Gamma(\gamma+\beta+\alpha+1)} t^{\alpha}
$$

for all $\beta>0$ and $t>0$; for the case $\alpha \geq 0$, the right hand side of (2.12) is less than or equal to $M t^{\alpha}$.

(ii) If, in addition to the assumption of $(i), \sigma(u) \leq 0$, then, for all $\lambda>0$,

$$
\left\|\mathfrak{a}_{\lambda}\right\| \leq M \frac{\Gamma(\gamma+\alpha+1)}{\Gamma(\gamma+1)} \lambda^{-\alpha}
$$

Proof. (i) Since the assumption implies

$$
k_{\gamma+1}(t)\left\|\mathfrak{c}_{t}^{\gamma}\right\| \leq \frac{t^{\gamma}}{\Gamma(\gamma+1)} M t^{\alpha}=M \frac{\Gamma(\gamma+\alpha+1)}{\Gamma(\gamma+1)} k_{\gamma+\alpha+1}(t)
$$

for all $t>0$, it follows that

$$
\begin{aligned}
\left\|\mathfrak{c}_{t}^{\gamma+\beta}\right\| & =\left\|\left(k_{\gamma+\beta+1}(t)\right)^{-1}\left(k_{\beta} *\left(k_{\gamma+1} \mathfrak{c}^{\gamma}\right)\right)(t)\right\| \quad(\mathrm{cf} . \text { the proof of Theorem 2.2(i) }) \\
& \leq M \frac{\Gamma(\gamma+\alpha+1)}{\Gamma(\gamma+1)} \frac{\Gamma(\gamma+\beta+1)}{t^{\gamma+\beta}}\left(k_{\beta} * k_{\gamma+\alpha+1}\right)(t) \\
& =M \frac{\Gamma(\gamma+\alpha+1)}{\Gamma(\gamma+1)} \frac{\Gamma(\gamma+\beta+1)}{t^{\gamma+\beta}} k_{\gamma+\beta+\alpha+1}(t) \\
& =M \frac{\Gamma(\gamma+\alpha+1)}{\Gamma(\gamma+1)} \frac{\Gamma(\gamma+\beta+1)}{\Gamma(\gamma+\beta+\alpha+1)} t^{\alpha}
\end{aligned}
$$


for all $t>0$.

Since $f(\cdot):=\ln \Gamma(\cdot)$ is a convex function on the interval $(0, \infty)$ (cf. e.g. [2], [13, p. 251]), for $\alpha, \beta, \gamma \geq 0$, we obtain

$$
\ln \left(\frac{\Gamma(\gamma+\alpha+1)}{\Gamma(\gamma+1)}\right) \leq \ln \left(\frac{\Gamma(\gamma+\beta+\alpha+1)}{\Gamma(\gamma+\beta+1)}\right) .
$$

Since $\ln (\cdot)$ is an increasing function, it follows that

$$
\frac{\Gamma(\gamma+\alpha+1)}{\Gamma(\gamma+1)} \frac{\Gamma(\gamma+\beta+1)}{\Gamma(\gamma+\beta+\alpha+1)} \leq 1 \quad(\alpha, \beta, \gamma \geq 0)
$$

which implies that the right hand side of (2.12) is less than or equal to $M t^{\alpha}$ when $\alpha \geq 0$.

(ii) Since $\sigma(u) \leq 0$, we can apply Theorem 2.3(ii) and then use $\left\|\mathfrak{c}_{t}^{\gamma}\right\| \leq M t^{\alpha}$ for all $t>0$ to obtain the following estimation for all $\lambda>0$ :

$$
\begin{aligned}
\left\|\mathfrak{a}_{\lambda}\right\| & =\lambda^{\gamma+1}\left\|\int_{0}^{\infty} e^{-\lambda t} k_{\gamma+1}(t) \mathfrak{c}_{t}^{\gamma} d t\right\| \leq \lambda^{\gamma+1} \int_{0}^{\infty} e^{-\lambda t} k_{\gamma+1}(t)\left\|\mathfrak{c}_{t}^{\gamma}\right\| d t \\
& =\lambda^{\gamma+1} M \frac{\Gamma(\gamma+\alpha+1)}{\Gamma(\gamma+1)} \int_{0}^{\infty} e^{-\lambda t} k_{\gamma+\alpha+1}(t) d t \\
& =\lambda^{\gamma+1} M \frac{\Gamma(\gamma+\alpha+1)}{\Gamma(\gamma+1)} \lambda^{-(\gamma+\alpha+1)}=M \frac{\Gamma(\gamma+\alpha+1)}{\Gamma(\gamma+1)} \lambda^{-\alpha} .
\end{aligned}
$$

Corollary 2.8. Let $\gamma \geq 0$ and $u \in C([0, \infty), X)$. Then the following hold.

(i) If $\alpha>-1-\gamma$ and $\left\|\mathfrak{c}_{t}^{\gamma}\right\|=O\left(t^{\alpha}\right)$ as $t \rightarrow \infty$, then $\left\|\mathfrak{c}_{t}^{\gamma^{\prime}}\right\|=O\left(t^{\alpha}\right)$ as $t \rightarrow \infty$ for all $\gamma^{\prime}>\gamma$; in addition, if $\sigma(u) \leq 0$, then $\left\|\mathfrak{a}_{\lambda}\right\|=O\left(\lambda^{-\alpha}\right)$ as $\lambda \downarrow 0$.

(ii) If $\gamma^{\prime}>\gamma$, then

$$
\alpha_{0}\left(\mathfrak{c}^{\gamma^{\prime}}\right) \leq \max \left\{\alpha_{0}\left(\mathfrak{c}^{\gamma}\right),-1-\gamma\right\}
$$

In addition, if $\sigma(u) \leq 0$, then

$$
\alpha_{0}(\mathfrak{a} .) \leq \max \left\{\alpha_{0}\left(\mathfrak{c}^{\gamma}\right),-1-\gamma\right\}
$$

Proof. (i) Let $\gamma^{\prime}>\gamma$. By the assumption we can choose two constants $M>0$ and $A>0$ so that $\left\|\mathfrak{c}_{t}^{\gamma}\right\| \leq M t^{\alpha}$ for all $t \geq A$. Then, putting $B=\left\|\mathfrak{c}_{t}^{\gamma} \chi_{[0, A]}(t)\right\|_{\infty}$ $(<\infty$ because $u$ is assumed to be continuous on $[0, \infty))$, we have

$$
\left\|\mathfrak{c}_{t}^{\gamma}\right\| \leq M t^{\alpha}+B \chi_{[0, A]}(t) \quad(t>0) .
$$


Noting the identity $\mathfrak{c}_{t}^{\gamma^{\prime}}=\left(k_{\gamma^{\prime}+1}(t)\right)^{-1}\left[k_{\gamma^{\prime}-\gamma} *\left(k_{\gamma+1} \mathfrak{c}^{\gamma}\right)\right](t)$, and using (2.16) together with the proof of Theorem 2.7(i), we have

$$
\begin{aligned}
\left\|\mathfrak{c}_{t}^{\gamma^{\prime}}\right\| \leq & \left(k_{\gamma^{\prime}+1}(t)^{-1}\left[k_{\gamma^{\prime}-\gamma} *\left(k_{\gamma+1}\left\|\mathfrak{c}^{\gamma}\right\|\right)\right](t)\right. \\
\leq & M \frac{\Gamma(\gamma+\alpha+1)}{\Gamma(\gamma+1)} \frac{\Gamma\left(\gamma^{\prime}+1\right)}{\Gamma\left(\gamma^{\prime}+\alpha+1\right)} t^{\alpha} \\
& +\frac{\Gamma\left(\gamma^{\prime}+1\right)}{t^{\gamma^{\prime}}} \int_{0}^{t} \frac{(t-s)^{\gamma^{\prime}-\gamma-1} s^{\gamma}}{\Gamma\left(\gamma^{\prime}-\gamma\right) \Gamma(\gamma+1)} B \chi_{[0, A]}(s) d s \\
\leq & M_{1} t^{\alpha}+\frac{\Gamma\left(\gamma^{\prime}+1\right)}{t^{\prime}} \frac{B A^{\gamma+1}}{\Gamma\left(\gamma^{\prime}-\gamma\right) \Gamma(\gamma+2)} t^{\gamma^{\prime}-\gamma}(t-A)^{-1} \\
\leq & M_{1} t^{\alpha}+\frac{2 \Gamma\left(\gamma^{\prime}+1\right) B A^{\gamma+1}}{\Gamma\left(\gamma^{\prime}-\gamma\right) \Gamma(\gamma+2)} t^{-\gamma-1}(\text { for all } t>2 A) .
\end{aligned}
$$

Since $\alpha>-1-\gamma$, this shows that $\left\|\mathfrak{c}_{t}^{\gamma^{\prime}}\right\|=O\left(t^{\alpha}\right)$ as $t \rightarrow \infty$.

Next, suppose $\sigma(u) \leq 0$. Using (2.16) together with the proof of Theorem 2.7(ii), we obtain

$$
\left\|\mathfrak{a}_{\lambda}\right\| \leq M \frac{\Gamma(\gamma+\alpha+1)}{\Gamma(\gamma+1)} \lambda^{-\alpha}+\lambda^{\gamma+1} B \int_{0}^{A} k_{\gamma+1}(t) d t
$$

for all $\lambda>0$, where

$$
B \int_{0}^{A}\left(k_{\gamma+1}\right)(t) d t=B k_{\gamma+2}(A)
$$

Since $\alpha+\gamma+1>0$ implies $\lambda^{-\alpha}>\lambda^{\gamma+1}$ for $0<\lambda<1$, it follows that

$$
\left\|\mathfrak{a}_{\lambda}\right\| \leq\left(M \frac{\Gamma(\gamma+\alpha+1)}{\Gamma(\gamma+1)}+B k_{\gamma+2}(A)\right) \lambda^{-\alpha} \quad(0<\lambda<1) .
$$

(ii) This is an immediate consequence of (i).

Remarks. (1) The existence of $M>0$ and $\alpha>-1-\gamma$ such that $\left\|\mathfrak{c}_{t}^{\gamma}\right\| \leq M t^{\alpha}$ for all $t>0$ does not imply $\sigma(u) \leq 0$, when $\gamma>1$. For example, the function $u$ as considered in the Remark under Theorem 2.3 satisfies $\left\|\mathfrak{c}_{t}^{2}\right\| \leq M$ for all $t>0$, but $\sigma(u)=1$. On the other hand, it follows from Theorems 2.2 and 2.3 that if $0 \leq \gamma \leq 1$ and $\left\|\mathfrak{c}_{t}^{\gamma}\right\|=O\left(t^{\alpha}\right)$ as $t \rightarrow \infty$, then $\sigma(u) \leq 0$.

(2) When $u \neq 0$ is a positive function in a Banach lattice $X$, the condition $\left\|\mathfrak{c}_{t}^{\gamma}\right\|=O\left(t^{\alpha}\right)$ as $t \rightarrow \infty$, where $\alpha \in \mathbb{R}$, does imply $\sigma(u) \leq 0$, and thus the assumption $\sigma(u) \leq 0$ can be omitted from Theorem 2.7 and Corollary 2.8. For, the 
condition $\left\|\mathfrak{c}_{t}^{\gamma}\right\|=O\left(t^{\alpha}\right)$ as $t \rightarrow \infty$ implies $w_{0}\left(\mathfrak{c}^{\gamma}\right) \leq 0$, and thus, by the Remark under Theorem 2.6,

$$
\sigma(u) \leq \max \{\sigma(u), 0\}=w_{0}\left(\mathfrak{c}^{\gamma+1}\right) \leq w_{0}\left(\mathfrak{c}^{\gamma}\right) \leq 0 .
$$

(3) Inequalities (2.14) and (2.15) are sharp. Here is an example:

Example 2. Define a positive function $u \in C([0, \infty), \mathbb{R})$ by $u(t):=\max \{1-$ $t, 0\}$ for $t \geq 0$. Then $w_{0}(u)=\alpha_{0}(u)=-\infty$; and by an easy calculation together with the binomial expansion $(1+t)^{\gamma+1}=1+\sum_{n=1}^{\infty}(\gamma+1) \gamma \ldots(\gamma+1-n+1) t^{n} / n$ ! for $t \in \mathbb{C}$ with $|t|<1$, we obtain that, for all $\gamma>0$ and $t>1$,

$$
\begin{gathered}
\mathfrak{c}_{t}^{\gamma}=\frac{\gamma}{t^{\gamma}} \int_{0}^{1}(t-s)^{\gamma-1}(1-s) d s=1+\frac{t}{\gamma+1}\left\{(1-1 / t)^{\gamma+1}-1\right\}, \\
=\frac{\gamma}{2} \frac{1}{t}(1+o(1)) \quad \text { as } t \rightarrow \infty,
\end{gathered}
$$

and

$$
\mathfrak{a}_{\lambda}=\lambda \int_{0}^{1} e^{-\lambda s}(1-s) d s=1-\left(1-e^{-\lambda}\right) / \lambda \text { for all } \lambda>0 .
$$

It follows that $w_{0}\left(\mathfrak{c}^{\gamma}\right)=0$ and $\alpha_{0}\left(\mathfrak{c}^{\gamma}\right)=-1$ for all $\gamma>0$. Since $\lim _{\lambda \downarrow 0} \lambda^{-1} \mathfrak{a}_{\lambda}=$ $\lim _{\lambda \downarrow 0} \lambda^{-1}-\left(1-e^{-\lambda}\right) \lambda^{-2}=2^{-1}$, it also follows that $\mathfrak{a}_{\lambda}=2^{-1} \lambda(1+o(1))$ as $\lambda \downarrow 0$, and hence $\alpha_{0}(\mathfrak{a})=$.-1 . Thus, both (2.14) and (2.15) become $\alpha_{0}\left(\mathfrak{c}^{\gamma^{\prime}}\right)=$ $\alpha_{0}(\mathfrak{a})=-1=.\max \left\{\alpha_{0}\left(\mathfrak{c}^{\gamma}\right),-1-\gamma\right\}$ for all $\gamma^{\prime}>\gamma \geq 0$.

(4) Let $\lambda_{0}>0$. Then the function $u_{1}(t):=t^{\lambda_{0}-1}$ for $t \geq 0$ satisfies

$$
\begin{aligned}
\mathfrak{c}_{t}^{\gamma}\left(u_{1}\right) & =\frac{\gamma}{t^{\gamma}} \int_{0}^{t}(t-s)^{\gamma-1} s^{\lambda_{0}-1} d s \\
& =\gamma t^{\lambda_{0}-1} \int_{0}^{1}(1-s)^{\gamma-1} s^{\lambda_{0}-1} d s=\gamma t^{\lambda_{0}-1} B\left(\gamma, \lambda_{0}\right)
\end{aligned}
$$

for all $\gamma>0$ and $t>0$. Further we have

$$
\mathfrak{a}_{\lambda}\left(u_{1}\right)=\lambda \int_{0}^{\infty} e^{-\lambda t} t^{\lambda_{0}-1} d t=\lambda^{-\left(\lambda_{0}-1\right)} \int_{0}^{\infty} e^{-t} t^{\lambda_{0}-1} d t=\lambda^{-\left(\lambda_{0}-1\right)} \Gamma\left(\lambda_{0}\right)
$$

for all $\lambda>0$. Thus we have $\alpha_{0}\left(\mathfrak{c}^{\gamma} \cdot\left(u_{1}\right)\right)=\alpha_{0}\left(u_{1}\right)=\lambda_{0}-1=\alpha_{0}\left(\mathfrak{a} \cdot\left(u_{1}\right)\right)$ for all $\gamma>0$. Of course this is a special case. In general the function $\gamma \mapsto \alpha_{0}\left(\boldsymbol{c}^{\gamma}\right)$ is not constant on $[0, \infty)$. To understand this situation, we give the following example.

Example 3. Define $u_{2}(t):=\chi_{[0,2 \pi]}(t) \sin t$ for $t \geq 0$. Then $\alpha_{0}\left(\mathfrak{a} .\left(u_{2}\right)\right)=$ $-2=\alpha_{0}\left(\mathfrak{c}^{\gamma} \cdot\left(u_{2}\right)\right)$ for all $\gamma \in[0, \infty) \backslash\{0,1\}$ and $\alpha_{0}\left(\mathfrak{c}^{0} \cdot\left(u_{2}\right)\right)=\alpha_{0}\left(\mathfrak{c}^{1} \cdot\left(u_{2}\right)\right)=-\infty$. 
To see this we first notice from the definition of $u_{2}$ that $\alpha_{0}\left(\mathfrak{c}^{0}\left(u_{2}\right)\right)=\alpha_{0}\left(\mathfrak{c}^{1} \cdot\left(u_{2}\right)\right)=$ $-\infty$. Next by an elementary calculation we obtain

$$
\mathfrak{a}_{\lambda}\left(u_{2}\right)=\frac{\lambda\left(1-e^{-2 \pi \lambda}\right)}{1+\lambda^{2}}
$$

for all $\lambda>0$. It follows that $\lim _{\lambda \downarrow 0} \lambda^{-2} \mathfrak{a}_{\lambda}=2 \pi$ and $\alpha_{0}\left(\mathfrak{a} .\left(u_{2}\right)\right)=-2$. Since $\alpha_{0}\left(\mathfrak{c}^{1} \cdot\left(u_{2}\right)\right)=-\infty$, it then follows from Corollary 2.8 that $\alpha_{0}\left(\mathfrak{c}^{\gamma} \cdot\left(u_{2}\right)\right)=-2$ for all $\gamma>1$. Finally, suppose $0<\gamma<1$. Then, for all $t>2 \pi$, we have

$$
\begin{aligned}
-\mathfrak{c}_{t}^{\gamma}\left(u_{2}\right) & =\frac{-\gamma}{t^{\gamma}} \int_{0}^{2 \pi}(t-s)^{\gamma-1} \sin s d s \\
& =\frac{-\gamma}{t^{\gamma}} \int_{0}^{\pi}\left((t-s)^{\gamma-1}-(t-\pi-s)^{\gamma-1}\right) \sin s d s \\
& \leq \frac{\gamma}{t^{\gamma}} \int_{0}^{\pi}\left\{(t-\pi-s)^{\gamma-1}-(t-s)^{\gamma-1}\right\} d s \\
& =\frac{1}{t^{\gamma}}\left\{2(t-\pi)^{\gamma}-(t-2 \pi)^{\gamma}-t^{\gamma}\right\}=2(1-\pi / t)^{\gamma}-(1-2 \pi / t)^{\gamma}-1 \\
& =-\gamma(\gamma-1) \pi^{2} \frac{1}{t^{2}}(1+o(1)) \quad \text { as } t \rightarrow \infty .
\end{aligned}
$$

Similarly, if $0<\delta<\pi / 2$, then

$$
\begin{aligned}
-\mathfrak{c}_{t}^{\gamma}\left(u_{2}\right) & \geq \frac{\gamma}{t^{\gamma}} \sin \delta \int_{\delta}^{\pi-\delta}\left\{(t-\pi-s)^{\gamma-1}-(t-s)^{\gamma-1}\right\} d s \\
& =\frac{\sin \delta}{t^{\gamma}}\left\{\left((t-\pi-\delta)^{\gamma}-(t-2 \pi+\delta)^{\gamma}\right)-\left((t-\delta)^{\gamma}-(t-\pi+\delta)^{\gamma}\right)\right\} \\
& =-\gamma(\gamma-1) \pi(\pi-2 \delta) \sin \delta \frac{1}{t^{2}}(1+o(1)) \quad \text { as } t \rightarrow \infty
\end{aligned}
$$

Hence it follows that $\alpha_{0}\left(\mathfrak{c}^{\gamma}\left(u_{2}\right)\right)=-2$ for all $0<\gamma<1$.

It is interesting to note that if $u_{3}(t):=\sin t$ for $t \geq 0$, then $\alpha_{0}\left(\mathfrak{a} \cdot\left(u_{3}\right)\right)=-1=$ $\alpha_{0}\left(\mathfrak{c}^{\gamma} \cdot\left(u_{3}\right)\right)$ for all $\gamma \geq 1$ and $\alpha_{0}\left(\mathfrak{c}^{\gamma} \cdot\left(u_{3}\right)\right)=-\gamma$ for all $0 \leq \gamma \leq 1$. This can be proved by a similar calculation.

Theorem 2.9. Let $X$ be a Banach lattice and $u \in C([0, \infty), X)$ be a positive $X$-valued function. Let $\gamma \geq 1$ and $\alpha>-1-\gamma$. Then

(i) $\sup _{t>0}\left\|t^{-\alpha} \mathfrak{c}_{t}^{\gamma}\right\|<\infty$ if and only if $\sigma(u) \leq 0$ and $\sup _{\lambda>0}\left\|\lambda^{\alpha} \mathfrak{a}_{\lambda}\right\|<\infty$;

(ii) $\left\|\mathfrak{c}_{t}^{\gamma}\right\|=O\left(t^{\alpha}\right)$ as $t \rightarrow \infty$ if and only if $\sigma(u) \leq 0$ and $\left\|\mathfrak{a}_{\lambda}\right\|=O\left(\lambda^{-\alpha}\right)$ as $\lambda \downarrow 0$. 
Proof. Each of the first conditions of (i) and (ii) implies $\sigma(u) \leq 0$, as was observed in the above Remark (2), so that the necessity parts of (i) and (ii) follow from Theorem 2.7(ii) and Corollary 2.8(i), respectively.

To show the sufficiency part of (i), suppose $\lambda^{\alpha}\left\|\mathfrak{a}_{\lambda}\right\| \leq M$ for all $\lambda>0$. By Theorem 2.3(ii) for all $\lambda>0$ we have

$$
\lambda^{\alpha} \mathfrak{a}_{\lambda}=\lambda^{\alpha+\gamma} \int_{0}^{\infty} e^{-\lambda s}\left(k_{\gamma-1} * u\right)(s) d s .
$$

Since $u$ is positive, it follows that

$$
\begin{aligned}
\lambda^{\alpha} \mathfrak{a}_{\lambda} & \geq \lambda^{\alpha+\gamma} \int_{0}^{t} e^{-\lambda s}\left(k_{\gamma-1} * u\right)(s) d s \\
& \geq \lambda^{\alpha+\gamma} e^{-\lambda t}\left(k_{\gamma} * u\right)(t)=\lambda^{\alpha+\gamma} e^{-\lambda t} k_{\gamma+1}(t) \mathfrak{c}_{t}^{\gamma} \geq 0 .
\end{aligned}
$$

Here, fix any $t>0$ and let $\lambda=1 / t$. Then we obtain that $t^{-\alpha}\left\|\mathfrak{c}_{t}^{\gamma}\right\| \leq M e \Gamma(\gamma+1)$ for all $t>0$, i.e., that $\sup _{t>0}\left\|t^{-\alpha} \mathfrak{c}_{t}^{\gamma}\right\|<\infty$. This proof also shows the sufficiency part of (ii), since $\lambda=1 / t \downarrow 0$ is equivalent to $t \rightarrow \infty$.

Corollary 2.10. Let $X$ be a Banach lattice and $u \in C([0, \infty), X)$ be a positive $X$-valued function. Then the following hold.

(i) If $\gamma \geq 1$ and $\alpha>-2$, then

$$
\sup _{t>0}\left\|t^{-\alpha} \mathfrak{c}_{t}^{\gamma}\right\|<\infty \Leftrightarrow \sup _{t>0}\left\|t^{-\alpha} \mathfrak{c}_{t}^{1}\right\|<\infty \Leftrightarrow \sigma(u) \leq 0 \text { and } \sup _{\lambda>0}\left\|\lambda^{\alpha} \mathfrak{a}_{\lambda}\right\|<\infty .
$$

(ii) If $u \neq 0$ and $\sigma(u) \leq 0$, then $\alpha_{0}\left(\mathfrak{c}^{\gamma}\right)=\alpha_{0}(\mathfrak{a}) \geq$.-1 for all $\gamma \geq 1$.

Proof. (i)This is direct from Theorem 2.9(i).

(ii) For all $\lambda>0$ we have

$$
\mathfrak{a}_{\lambda}=\lambda \int_{0}^{\infty} e^{-\lambda t} u(t) d t \geq \lambda \int_{0}^{K} e^{-\lambda t} u(t) d t \geq \lambda e^{-\lambda K} \int_{0}^{K} u(t) d t \geq 0,
$$

and the hypothesis $u \neq 0$ implies

$$
\lim _{\lambda \downarrow 0} e^{-\lambda K} \int_{0}^{K} u(t) d t=\int_{0}^{K} u(t) d t>0
$$

for some $K>0$. Thus it follows that $\alpha_{0}(\mathfrak{a}$. $) \geq-1$. By this and Theorem 2.9(ii), we see that $\alpha_{0}\left(\mathfrak{c}^{\gamma}\right)=\alpha_{0}(\mathfrak{a}$. $) \geq-1$ for all $\gamma \geq 1$. 
Remark. If the positivity of $u \in C([0, \infty), X)$ is not assumed, then Theorem 2.9 and Corollary 2.10 do not hold. This can be seen in Section 3. Further, we note that the hypothesis $\gamma \geq 1$ is essential in Theorem 2.9 and Corollary 2.10. This can be seen in Section 4.

\section{Growth Orders of Means of $C_{0}$-Semigroups}

In this section we consider the case $u(t)=T(t) x$, where $(T(t))_{t \geq 0}$ is a $C_{0^{-}}$ semigroup of bounded linear operators on a Banach space $X$ and $x \in X$. We recall that in this case the notations $C_{t}^{\gamma} x$ and $A_{\lambda} x$ are used instead of $\mathfrak{c}_{t}^{\gamma}(T(\cdot) x)$ and $\mathfrak{a}_{\lambda}(T(\cdot) x)$, respectively. Further we use the notations $C_{t}^{\gamma}$ and $A_{\lambda}$ to denote bounded linear operators on $X$ defined as $C_{t}^{0}:=T(t)(t \geq 0), C_{0}^{\gamma}:=T(0)$, and for $\gamma, t>0, x \in X$,

$$
C_{t}^{\gamma} x=\gamma t^{-\gamma}\left(\int_{0}^{t}(t-s)^{\gamma-1} T(s) d s\right) x:=\gamma t^{-\gamma} \int_{0}^{t}(t-s)^{\gamma-1} T(s) x d s,
$$

and for $\lambda \in \mathbb{C}$ with $\operatorname{Re} \lambda>0$,

$$
A_{\lambda} x=\lambda\left(\int_{0}^{\infty} e^{-\lambda s} T(s) d s\right) x:=\lambda \lim _{t \rightarrow \infty} \int_{0}^{t} e^{-\lambda s} T(s) x d s \quad(x \in X)
$$

if the limit exists for all $x \in X$. The abscissa of convergence $\sigma(T(\cdot))$ of the Laplace integral $\left(\int_{0}^{\infty} e^{-\lambda s} T(s) d s\right) x:=\lim _{t \rightarrow \infty} \int_{0}^{t} e^{-\lambda s} T(s) x d s$ of $(T(t))_{t \geq 0}$ is defined as

$$
\begin{aligned}
\sigma(T(\cdot)) & :=\inf \left\{\operatorname{Re} \lambda: \lim _{t \rightarrow \infty} \int_{0}^{t} e^{-\lambda s} T(s) x d s \text { exists for all } x \in X\right\} \\
& =\sup \{\sigma(T(\cdot) x): x \in X\}
\end{aligned}
$$

It follows from the uniform boundedness principle that $\|T(t)\|=O\left(e^{w t}\right)$ as $t \rightarrow \infty$ if and only if $\|T(t) x\|=O\left(e^{w t}\right)$ as $t \rightarrow \infty$ for all $x \in X$, whence

$$
w_{0}(T(\cdot))=\sup \left\{w_{0}(T(\cdot) x): x \in X\right\} .
$$

Similarly, $\|T(t)\|=O\left(t^{\alpha}\right)$ as $t \rightarrow \infty$ if and only if $\|T(t) x\|=O\left(t^{\alpha}\right)$ as $t \rightarrow \infty$ for all $x \in X$; and

$$
\alpha_{0}(T(\cdot))=\sup \left\{\alpha_{0}(T(\cdot) x): x \in X\right\} .
$$

If $\sigma(T(\cdot)) \leq 0$, then $\left\|A_{\lambda}\right\|=O\left(\lambda^{-\alpha}\right)$ as $\lambda \downarrow 0$ if and only if $\left\|A_{\lambda} x\right\|=O\left(\lambda^{-\alpha}\right)$ as $\lambda \downarrow 0$ for all $x \in X$; and

$$
\alpha_{0}(A .)=\sup \left\{\alpha_{0}(A . x): x \in X\right\} .
$$


It is clear that $\sigma(T(\cdot)) \leq w_{0}(T(\cdot))$. We also note (see e.g. [1, Section 5.1]) that if $\lambda \in \mathbb{C}$ satisfies $\operatorname{Re} \lambda>\sigma(T(\cdot))$, then $\lambda-A$ is invertible and $(\lambda-A)^{-1}$ is a bounded operator on $X$ (i.e. $\lambda \in \rho(A)$ ), and $(\lambda-A)^{-1}=\int_{0}^{\infty} e^{-\lambda s} T(s) d s$, where $A$ and $\rho(A)$ denote the generator of $(T(t))_{t \geq 0}$ and the resolvent set of $A$, respectively. Thus if $\operatorname{Re} \lambda>\max \{\sigma(T(\cdot)), 0\}$, then $\lambda(\lambda-A)^{-1}=A_{\lambda}$. We mainly consider $A_{\lambda}$ with $\lambda \in \mathbb{R}$ such that $\lambda>\max \{\sigma(T(\cdot)), 0\}$.

We are interested in the question when one of the inequalities in (5) becomes an equality and for what examples it is a strict inequality. First, a consequence of Theorem 2.2(ii) and Corollary 2.8(ii) is stated as follows.

Theorem 3.1. The following hold for all $0 \leq \gamma<\gamma^{\prime}<\infty$.

(i) $\max \left\{w_{0}\left(C^{\gamma^{\prime}}\right), 0\right\} \leq \max \left\{w_{0}\left(C^{\gamma}\right), 0\right\}$.

(ii) $\alpha_{0}\left(C^{\gamma^{\prime}}\right) \leq \max \left\{\alpha_{0}\left(C^{\gamma}\right),-1-\gamma\right\}$; in addition, if $\sigma(T(\cdot)) \leq 0$, then $\alpha_{0}(A$. $) \leq$ $\max \left\{\alpha_{0}\left(C^{\gamma}\right),-1-\gamma\right\}$.

Remark. By Theorem 2.4, together with the uniform boundedness principle, (i) if $\sigma(T(\cdot))=0$, then $\sup _{\lambda>w}\left\|A_{\lambda}\right\|=\sup _{\lambda>w}\left\|\lambda(\lambda-A)^{-1}\right\|<\infty$ for all $w>0$; (ii) if $\sigma(T(\cdot))<0$, then $\sup _{\lambda>0}\left\|(1+\lambda)(\lambda-A)^{-1}\right\|<\infty$. As for (i) we note that the inequality $\sup _{\lambda>w}\left\|A_{\lambda}\right\|<\infty$ may fail to hold when $w>0$ is repalced with $w=0$. A counterexample can be found in the proof of Proposition 2.5 of [8].

Theorem 3.2. Suppose $\operatorname{dim} X=2$. Then for a $C_{0}$-semigroup $(T(t))_{t \geq 0}$ on $X$ such that $\sigma(T(\cdot)) \leq 0$, the following hold.

(i) $\|T(t)\|=O(t)$ as $t \rightarrow \infty,\left\|C_{t}^{1}\right\|=O(t)$ as $t \rightarrow \infty$, and $\left\|A_{\lambda}\right\|=O\left(\lambda^{-1}\right)$ as $\lambda \downarrow 0$.

(ii) If $0 \leq \alpha<1$, then $\left\|C_{t}^{1}\right\|=O\left(t^{\alpha}\right)$ as $t \rightarrow \infty$ is equivalent to $\left\|A_{\lambda}\right\|=$ $O\left(\lambda^{-\alpha}\right)$ as $\lambda \downarrow 0$, which is also equivalent to $\left\|C_{t}^{\gamma}\right\|=O\left(t^{\alpha}\right)$ as $t \rightarrow \infty$ for any $\gamma>1$. Hence, in particular, $(T(t))_{t \geq 0}$ is Abel-mean-bounded if and only if it is $\gamma$-Cesàro-mean-bounded for any $\gamma \geq 1$.

Proof. $\quad$ Since $\operatorname{dim} X=2$ implies $w_{0}(T(\cdot))=\sigma(T(\cdot)) \leq 0$, (i) and the first part of (ii) have been proved in Proposition 2.5 of [8]. Also it follows from Corollary 2.8(i) that, for all $\gamma>1$,

$$
\left\|C_{t}^{1}\right\|=O\left(t^{\alpha}\right)(t \rightarrow \infty) \Rightarrow\left\|C_{t}^{\gamma}\right\|=O\left(t^{\alpha}\right)(t \rightarrow \infty),
$$

and

$$
\left\|C_{t}^{\gamma}\right\|=O\left(t^{\alpha}\right)(t \rightarrow \infty) \Rightarrow\left\|A_{\lambda}\right\|=O\left(\lambda^{-\alpha}\right)(\lambda \downarrow 0) .
$$

Hence the proof is complete.

Remark. It is interesting to note that if $\operatorname{dim} X=1$, or $(T(t))_{t \geq 0}$ is an eventually norm-continuous $C_{0}$-semigroup of normal operators on a Hilbert space, or $(T(t))_{t \geq 0}$ 
is an eventually norm-continuous $C_{0}$-semigroup of hermitian operators on a Banach space, then $\sigma(T(\cdot)) \leq 0$ is equivalent to $\|T(t)\| \leq 1$ for all $t \geq 0$. This follows from the fact that, in each of the above cases, $w_{0}(T(\cdot))=\sigma(T(\cdot)) \leq 0$ and $\|T(t)\|=r(T(t))=e^{t w_{0}(T(\cdot))}$ for all $t \geq 0$. (See Corollary 2.2 of [8].)

Theorem 3.3. Let $(T(t))_{t \geq 0}$ be a $C_{0}$-semigroup on $X$ with generator $A$ and let $\gamma \geq 0$. Then the following hold.

(i) If $A$ is bounded, then $\max \left\{w_{0}\left(C^{\gamma+1}\right), 0\right\}=\max \left\{w_{0}\left(C^{\gamma}{ }^{\gamma}\right), 0\right\}, \alpha_{0}\left(C^{\gamma}\right) \leq$ $\max \left\{\alpha_{0}\left(C^{\gamma+1}\right)+1,0\right\}$, and $\alpha_{0}\left(C^{\gamma+1}\right) \geq \max \left\{\alpha_{0}\left(C^{\gamma}\right), 0\right\}-1$.

(ii) If $0 \in \rho(A)$, then $\alpha_{0}\left(C^{\gamma+1}\right) \leq \max \left\{\alpha_{0}\left(C^{\gamma}\right), 0\right\}-1$.

(iii) If $A$ is bounded and $0 \in \rho(A)$, then

$$
\begin{aligned}
& (\gamma+1)\|A\|^{-1}\left\|C_{t}^{\gamma}-I\right\| t^{-1} \leq\left\|C_{t}^{\gamma+1}\right\| \leq(\gamma+1)\left\|A^{-1}\right\|\left\|C_{t}^{\gamma}-I\right\| t^{-1} \quad(t>0), \\
& \text { and }
\end{aligned}
$$

$$
\alpha_{0}\left(C^{\gamma+1}\right)=\max \left\{\alpha_{0}\left(C^{\gamma}\right), 0\right\}-1
$$

Proof. We first show the following identity for all $\gamma \geq 0$ :

$$
A C_{t}^{\gamma+1}=(\gamma+1) t^{-1}\left(C_{t}^{\gamma}-I\right), t>0
$$

The case $\gamma=0$ is trivial. Now suppose $\gamma>0$. Then, since $A$ is a closed operator, we have, for all $x \in X$,

$$
\begin{aligned}
A C_{t}^{\gamma+1} x & =(\gamma+1) t^{-\gamma-1} A \int_{0}^{t}(t-s)^{\gamma} T(s) x d s \\
& =(\gamma+1) t^{-\gamma-1} A \int_{0}^{t}(t-s)^{\gamma} d\left(\int_{0}^{s} T(u) x d u\right) d s \\
& =(\gamma+1) t^{-\gamma-1} A \int_{0}^{t} \gamma(t-s)^{\gamma-1}\left(\int_{0}^{s} T(u) x d u\right) d s \\
& =(\gamma+1) t^{-\gamma-1} \int_{0}^{t} \gamma(t-s)^{\gamma-1}(T(s)-I) x d s \\
& =(\gamma+1) t^{-1} \gamma t^{-\gamma} \int_{0}^{t}(t-s)^{\gamma-1} T(s) x d s-(\gamma+1) t^{-1} x \\
& =(\gamma+1) t^{-1} C_{t}^{\gamma} x-(\gamma+1) t^{-1} x
\end{aligned}
$$


(i) If $A=0$, then $T(t)=I=C_{t}^{\beta}$ for all $t, \beta \geq 0$, so that $w_{0}\left(C^{\beta}\right)=0$ and $\alpha_{0}\left(C^{\beta}\right)=0$ for all $\beta \geq 0$. Next, suppose $A \neq 0$ is bounded. Then for all $t>0$ we have

$$
(\gamma+1)\left\|C_{t}^{\gamma}\right\| \leq t\|A\|\left\|C_{t}^{\gamma+1}\right\|+(\gamma+1)
$$

so that $\max \left\{w_{0}\left(C^{\gamma}\right), 0\right\} \leq \max \left\{w_{0}\left(C^{\gamma+1}\right), 0\right\} \leq \max \left\{w_{0}\left(C^{\gamma}\right), 0\right\}$ (Theorem 3.1(i)), and $\alpha_{0}\left(C^{\gamma}\right) \leq \max \left\{\alpha_{0}\left(C^{\gamma+1}\right)+1,0\right\}$. Also from the inequality

$$
\|A\|\left\|C_{t}^{\gamma+1}\right\| \geq(\gamma+1) t^{-1}\left\|C_{t}^{\gamma}-I\right\|, t>0
$$

it follows that $\alpha_{0}\left(C^{\gamma+1}\right)+1 \geq \alpha_{0}\left(C^{\gamma}-I\right)=\max \left\{\alpha_{0}\left(C^{\gamma}{ }^{\gamma}\right), 0\right\}$.

(ii) If $0 \in \rho(A)$, then

$$
\left\|C_{t}^{\gamma+1}\right\| \leq(\gamma+1)\left\|A^{-1}\right\|\left\|C_{t}^{\gamma}-I\right\| t^{-1}, t>0,
$$

and so $\alpha_{0}\left(C^{\gamma+1}\right) \leq \max \left\{\alpha_{0}\left(C .^{\gamma}\right), 0\right\}-1$.

(iii) This follows immediately from the above proofs of (i) and (ii).

Theorem 3.4. Let $k \geq 1$ be an integer, and let $(T(t))_{t \geq 0}$ be a $C_{0}$-semigroup on $X$ with generator $A=$ ai $I+N$, where $N$ is a bounded nilpotent operator on $X$ of order $k+1$ (i.e., $N^{k} \neq 0$ and $N^{k+1}=0$ ) and $a \in \mathbb{R}$. If $a \neq 0$, then the following hold.

(i) $\left\|C_{t}^{n}\right\| \sim t^{k-n}(t \rightarrow \infty)$ for $n=0,1, \ldots, k$, and $\left\|C_{t}^{n}\right\| \sim t^{-1}(t \rightarrow \infty)$ for all integers $n \geq k+1$. Therefore

$$
\alpha_{0}\left(C_{.}^{n}\right)= \begin{cases}k-n & \text { for } n=0,1, \ldots, k \\ -1 & \text { for all integers } n \geq k+1 .\end{cases}
$$

(ii) $\left\|A_{\lambda}\right\| \sim \lambda(\lambda \downarrow 0)$, and $\sup _{\lambda>0}\left\|A_{\lambda}\right\|<\infty$. In particular, $\alpha_{0}(A)=$.-1 .

Proof. (i) Since $C_{t}^{0}=T(t)=e^{t A}=e^{a i t} e^{t N}=e^{a i t}\left(\sum_{n=0}^{k}\left(t^{n} / n !\right) N^{n}\right)$, we have

$$
\sum_{n=0}^{k} \frac{t^{n}}{n !}\left\|N^{n}\right\| \geq\left\|C_{t}^{0}\right\| \geq \frac{t^{k}}{k !}\left\|N^{k}\right\|-\sum_{n=0}^{k-1} \frac{t^{n}}{n !}\left\|N^{n}\right\| .
$$

Therefore

$$
\lim _{t \rightarrow \infty} \frac{\left\|C_{t}^{0}\right\|}{t^{k}}=\frac{1}{k !}\left\|N^{k}\right\|>0
$$


Hence $\left\|C_{t}^{0}\right\| \sim t^{k}(t \rightarrow \infty)$, and $\alpha_{0}\left(C^{0}\right)=k$. Since $A$ is bounded and $0 \in \rho(A)$ (because $0 \neq a \in \mathbb{R}$ ), it follows from Theorem 3.3(iii) together with an induction argument that $\left\|C_{t}^{n}\right\| \sim t^{k-n}(t \rightarrow \infty)$ and $\alpha_{0}\left(C^{n}\right)=k-n$ for all $n=0,1, \ldots, k$.

To see that $\left\|C_{t}^{k+1}\right\| \sim t^{-1}(t \rightarrow \infty)$, take $x \in X$ such that $\|x\|=1$ and $N^{k} x \neq 0$. Then the set $\left\{x, N x, \ldots, N^{k} x\right\}$ is lenearly independent. To show this, suppose that $\sum_{j=0}^{k} b_{j} N^{j} x=0$. Then, since $N^{k+1}=0$, it follows that $b_{0} N^{k} x=N^{k}\left(\sum_{j=0}^{k} b_{j} N^{j} x\right)=0$, and hence $b_{0}=0$. Next we have $b_{1} N^{k} x=$ $N^{k-1}\left(\sum_{j=1}^{k} b_{j} N^{j} x\right)=0$, and hence $b_{1}=0$. By continuing this process, it follows that $b_{0}=b_{1}=\ldots=b_{k}=0$, whence the set $\left\{x, N x, \ldots, N^{k} x\right\}$ is linearly independent. Now, for all $\gamma>0$ and $t>0$, we have

$$
\begin{gathered}
C_{t}^{\gamma} x=\gamma t^{-\gamma} \int_{0}^{t}(t-s)^{\gamma-1} T(s) x d s=\gamma t^{-\gamma}\left(\int_{0}^{t}(t-s)^{\gamma-1} e^{a i s} x d s\right) \\
\quad+\gamma t^{-\gamma} \sum_{n=1}^{k}\left(\int_{0}^{t}(t-s)^{\gamma-1} \frac{e^{a i s} s^{n}}{n !} N^{n} x d s\right) .
\end{gathered}
$$

Since $0 \neq a \in \mathbb{R}$, it follows that $\lim _{t \rightarrow \infty} \gamma t^{-\gamma}\left(\int_{0}^{t}(t-s)^{\gamma-1} e^{a i s} x d s\right)=0$. Thus

$$
\liminf _{t \rightarrow \infty}\left\|C_{t}^{\gamma}-I\right\| \geq \liminf _{t \rightarrow \infty}\left\|C_{t}^{\gamma} x-x\right\|>0 .
$$

Using this for $\gamma=k$, together with the fact that $\left\|C_{t}^{k}\right\| \sim t^{0}(t \rightarrow \infty)$ (so that $\lim \sup _{t \rightarrow \infty}\left\|C_{t}^{k}-I\right\|<\infty$ ) and Theorem 3.3(iii), we obtain

$$
\left\|C_{t}^{k+1}\right\| \sim t^{-1}(t \rightarrow \infty), \quad \text { and } \quad \alpha_{0}\left(C^{k+1}\right)=-1 .
$$

It is now obvious from Theorem 3.3(iii) and an induction argument that $\left\|C_{t}^{n}\right\| \sim$ $t^{-1}(t \rightarrow \infty)$ and $\alpha_{0}\left(C^{n}\right)=-1$ for all integers $n>k+1$.

(ii) We have

$$
\begin{aligned}
\lim _{\lambda \downarrow 0}\left\|A_{\lambda}\right\| / \lambda & =\lim _{\lambda \downarrow 0}\left\|\int_{0}^{\infty} e^{-\lambda t} e^{a i t}\left(\sum_{n=0}^{k} \frac{t^{n}}{n !} N^{n}\right) d t\right\| \\
& =\lim _{\lambda \downarrow 0}\left\|\sum_{n=0}^{k} \frac{1}{(\lambda-a i)^{n+1}} N^{n}\right\|=\frac{1}{|a|}\left\|\sum_{n=0}^{k}\left(\frac{N}{-a i}\right)^{n}\right\|,
\end{aligned}
$$

which is positive because

$$
\sum_{n=0}^{k}\left(\frac{N}{-a i}\right)^{n}\left(I+\frac{N}{a i}\right)=I-\left(\frac{N}{-a i}\right)^{k+1}=I .
$$


Hence $\left\|A_{\lambda}\right\| \sim \lambda(\lambda \downarrow 0)$, and thus $\alpha_{0}\left(A_{\lambda}\right)=-1$. It is direct from the above calculation that $\sup _{\lambda>0}\left\|A_{\lambda}\right\|<\infty$. (This also follows from Theorem 2.7(ii) since $\sup _{t>0}\left\|C_{t}^{k}\right\|<\infty$.)

When $k \geq 1$, it follows from Theorem 3.4 and Corollary 2.8 that, for the $C_{0}$-semigroup $(T(t))_{t \geq 0}$ in Theorem 3.4, $\alpha_{0}\left(C^{\gamma}{ }^{\gamma}\right)$ is a non-increasing function of $\gamma \in[0, \infty)$ and satisfies

$$
\begin{aligned}
\alpha_{0}\left(C_{.}^{0}\right)=k & >\alpha_{0}\left(C_{.}^{1}\right)=k-1>\cdots>\alpha_{0}\left(C_{.}^{k}\right)=0 \\
& >-1=\alpha_{0}\left(C_{.}^{\gamma}\right)=\alpha_{0}(A .)
\end{aligned}
$$

for all $\gamma \geq k+1$.

It also follows (cf. Theorem 3.4(i), Theorem 2.2(i)) that

(3.10) $\lim _{t \rightarrow \infty}\left\|C_{t}^{\gamma}\right\|=\infty$ for all $\gamma \in[0, k-1], \sup _{t>0}\left\|C_{t}^{\gamma}\right\|<\infty$ for all $\gamma \in[k, k+1)$,

and $\quad\left\|C_{t}^{\gamma}\right\| \sim t^{-1}(t \rightarrow \infty)$ for all $\gamma \geq k+1$.

So far the situation on the interval $(k-1, k)$ is not clear. We will see from the proof of the following theorem that $\sup _{t>0}\left\|C_{t}^{\gamma}\right\|=\infty$ for all $\gamma \in(k-1, k)$.

Theorem 3.5. Let $k \in \mathbb{N}$. Then there exists a $C_{0}$-semigroup $(T(t))_{t \geq 0}$ [resp. a strongly continuous cosine operator function $(C(t))_{t \geq 0}$ ] of bounded linear operators on $X$ such that $\sup _{t>0}\left\|C_{t}^{k}\right\|<\infty$, but $\sup _{t>0}\left\|C_{t}^{\gamma}\right\|=\infty$ for all $\gamma$ with $0 \leq \gamma<k$.

We need first to prove the following key lemma.

Lemma 3.6. Let $0<\gamma<1$. Then for every integer $k \geq 0$ we have

$$
\lim _{n \rightarrow \infty} n \int_{0}^{1}(1-s)^{\gamma-1} s^{k} \cos (2 \pi n s) d s=\infty
$$

and

$$
\lim _{n \rightarrow \infty} n \int_{0}^{1}(1-s)^{\gamma-1} s^{k} \sin (2 \pi n s) d s=-\infty
$$

Proof. For simplicity we set, for $n \geq 0$,

(3.13) $a_{n}:=\int_{0}^{1}(1-s)^{\gamma-1} s^{k} \cos (2 \pi n s) d s, \quad b_{n}:=\int_{0}^{1}(1-s)^{\gamma-1} s^{k} \sin (2 \pi n s) d s$; 
and

$$
f(s):=s^{\gamma-1}(1-s)^{k} \quad(s \in[0,1]) .
$$

We first prove that $a_{n}>0>b_{n}$ for all $n \geq 1$. By a change of variable we have $a_{n}=\int_{0}^{1} s^{\gamma-1}(1-s)^{k} \cos (2 \pi n s) d s$, and $-b_{n}=\int_{0}^{1} s^{\gamma-1}(1-s)^{k} \sin (2 \pi n s) d s$, whence

$$
a_{n}=\sum_{j=1}^{n} \int_{(j-1) / n}^{j / n} f(s) \cos (2 \pi n s) d s \quad \text { (by (3.14)) }
$$

and furthermore

$$
\begin{aligned}
\int_{0}^{1 / n} f(s) \cos (2 \pi n s) d s= & \int_{0}^{1 / 4 n}\{f(s)-f((1 / 2 n)+s)\} \cos (2 \pi n s) d s \\
& \left.-\int_{0}^{1 / 4 n}\{f((1 / 2 n)-s)-f(1 / n)-s)\right\} \cos (2 \pi n s) d s \\
= & \int_{0}^{1 / 4 n}\{\{f(s)-f((1 / 2 n)+s)\}-\{f((1 / 2 n)-s) \\
& -f((1 / 2 n)+(1 / 2 n)-s)\}\} \cos (2 \pi n s) d s .
\end{aligned}
$$

Since $f$ is positive, strictly decreasing and convex on the interval $(0,1)$, if $0<s<$ $1 / 4 n$ then we have

$$
\{\{f(s)-f((1 / 2 n)+s)\}-\{f((1 / 2 n)-s)-f((1 / 2 n)+(1 / 2 n)-s)\}\}>0 .
$$

Since $\cos (2 \pi n s)>0$ on the interval $(0,1 / 4 n)$, we conclude that

$$
\int_{0}^{1 / n} f(s) \cos (2 \pi n s) d s>0 .
$$

By the same argument we see that $\int_{(i-1) / n}^{i / n} f(s) \cos (2 \pi n s) d s>0$ for each $1 \leq$ $i \leq n$. Consequently, $a_{n}>0$ for all $n \geq 1$. It is similar and easier to prove that $-\bar{b}_{n}=\int_{0}^{1} f(s) \sin (2 \pi n s) d s>0$ for all $n \geq 1$; hence we may omit the proof.

We next prove that

$$
\int_{1 / 8 n}^{1-(7 / 8 n)} f(s) \sin (2 \pi n s) d s>0 \quad(n \geq 1) .
$$

To do this, write 


$$
\int_{1 / 8 n}^{1-(7 / 8 n)} f(s) \sin (2 \pi n s) d s=\sum_{j=1}^{n-1} \int_{(j-1) / n+1 / 8 n}^{j / n+1 / 8 n} f(s) \sin (2 \pi n s) d s .
$$

By an elementary calculation

$$
\begin{aligned}
& \int_{1 / 8 n}^{1 / n+1 / 8 n} f(s) \sin (2 \pi n s) d s \\
= & \int_{0}^{3 / 8 n}\{f((1 / 2 n)-s)-f((1 / 2 n)+s)\} \sin (2 \pi n s) d s \\
& -\int_{0}^{1 / 8 n}\{f((1 / n)-s)-f((1 / n)+s)\} \sin (2 \pi n s) d s \\
= & \int_{0}^{1 / 8 n}\{\{f((1 / 2 n)-s)-f((1 / 2 n)+s)\}-\{f((1 / n)-s) \\
& -f((1 / n)+s)\}\} \sin (2 \pi n s) d s \\
& +\int_{1 / 8 n}^{3 / 8 n}\{f((1 / 2 n)-s)-f((1 / 2 n)+s)\} \sin (2 \pi n s) d s .
\end{aligned}
$$

As before, if $0<s<1 / 8 n$, then

$\{\{f((1 / 2 n)-s)-f((1 / 2 n)+s)\}-\{f((1 / n)-s)-f((1 / n)+s)\}\} \sin (2 \pi n s)>0$.

On the other hand, it is immediate that if $1 / 8 n<s<3 / 8 n$, then

$$
\{f((1 / 2 n)-s)-f((1 / 2 n)+s)\} \sin (2 \pi n s)>0 .
$$

Therefore it follows that

$$
\int_{1 / 8 n}^{1 / n+1 / 8 n} f(s) \sin (2 \pi n s) d s>0 .
$$

By the same argument we have

$$
\int_{(j-1) / n+1 / 8 n}^{j / n+1 / 8 n} f(s) \sin (2 \pi n s) d s>0 \quad(1 \leq j \leq n-1) .
$$

This proves (3.15). We then note that

$$
\begin{aligned}
\int_{0}^{1 / 8 n} f(s) \sin (2 \pi n s) d s & =\int_{0}^{1 / 8 n} s^{\gamma-1}(1-s)^{k} \sin (2 \pi n s) d s \\
& \geq\left(\frac{8 n-1}{8 n}\right)^{k} \frac{2}{\pi} \int_{0}^{1 / 8 n} s^{\gamma-1}(2 \pi n s) d s \\
& =\left(\frac{8 n-1}{8 n}\right)^{k} 4 n \int_{0}^{1 / 8 n} s^{\gamma} d s \\
& =(1-(1 / 8 n))^{k} \frac{4}{(\gamma+1) 8^{\gamma+1}} n^{-\gamma} .
\end{aligned}
$$


Here, since $\lim _{s \uparrow 1} f(s)=1$ (when $k=0$ ) or 0 (when $k \geq 1$ ), it follows that

$$
\left|\int_{1-(7 / 8 n)}^{1} f(s) \sin (2 \pi n s) d s\right| \leq \int_{1-(7 / 8 n)}^{1} f(s) d s=n^{-1} \cdot O(1)=n^{-\gamma} \cdot o(1),
$$

so that, combining (3.15) and (3.16), we obtain

$$
\begin{aligned}
(-n) b_{n} & =n \int_{0}^{1} f(s) \sin (2 \pi n s) d s \\
& =n\left(\int_{0}^{1 / 8 n}+\int_{1 / 8 n}^{1-(7 / 8 n)}+\int_{1-(7 / 8 n)}^{1}\right) f(s) \sin (2 \pi n s) d s \\
& >n\left(\int_{0}^{1 / 8 n}+\int_{1-(7 / 8 n)}^{1}\right) f(s) \sin (2 \pi n s) d s \longrightarrow \infty \text { as } n \rightarrow \infty .
\end{aligned}
$$

This proves (3.12).

To prove (3.11), we note that

$$
a_{n}=\int_{0}^{1} f(s) \cos (2 \pi n s) d s>\int_{0}^{1 / n} f(s) \cos (2 \pi n s) d s>0,
$$

and that there exists a unique constant $c_{n}$, with $0<c_{n}<1 / 4 n$, such that

$$
\int_{c_{n}}^{1 / n} f(s) \cos (2 \pi n s) d s=0 .
$$

Write

$$
I(n):=\int_{1 / 2 n}^{1 / n} f(s) \cos (2 \pi n s) d s .
$$

Since $f$ is positive and strictly decreasing on the interval $[0,1]$, it follows that

$$
I(n)=\frac{1}{n} \int_{1 / 2}^{1} f(s / n) \cos (2 \pi s) d s=n^{-\gamma} \int_{1 / 2}^{1} s^{\gamma-1}(1-s / n)^{k} \cos (2 \pi s) d s<0,
$$

and by (3.18)

$$
0<-I(n)=\int_{c_{n}}^{1 / 2 n} f(s) \cos (2 \pi n s) d s=n^{-\gamma} \int_{n c_{n}}^{1 / 2} s^{\gamma-1}(1-s / n)^{k} \cos (2 \pi s) d s .
$$

Using these facts we obtain

$$
0<-\int_{1 / 2}^{1} s^{\gamma-1}(1-s / n)^{k} \cos (2 \pi s) d s=\int_{n c_{n}}^{1 / 2} s^{\gamma-1}(1-s / n)^{k} \cos (2 \pi s) d s
$$


for all $n \geq 1$. Now, by Lebesgue's convergence theorem,

$$
\lim _{n \rightarrow \infty} \int_{1 / 2}^{1} s^{\gamma-1}(1-s / n)^{k} \cos (2 \pi s) d s=\int_{1 / 2}^{1} s^{\gamma-1} \cos (2 \pi s) d s<0 .
$$

Thus

$$
\lim _{n \rightarrow \infty} \int_{n c_{n}}^{1 / 2} s^{\gamma-1}(1-s / n)^{k} \cos (2 \pi s) d s=-\int_{1 / 2}^{1} s^{\gamma-1} \cos (2 \pi s) d s>0 ;
$$

and since $\int_{0}^{1} s^{\gamma-1} \cos (2 \pi s) d s>0$ and $\cos (2 \pi s)>0$ on the interval $(0,1 / 4)$, there exists a unique constant $c$, with $0<c<1 / 4$, such that

$$
\int_{c}^{1 / 2} s^{\gamma-1} \cos (2 \pi s) d s=-\int_{1 / 2}^{1} s^{\gamma-1} \cos (2 \pi s) d s .
$$

Therefore we have

$$
\lim _{n \rightarrow \infty} \int_{n c_{n}}^{1 / 2} s^{\gamma-1}(1-s / n)^{k} \cos (2 \pi s) d s=\int_{c}^{1 / 2} s^{\gamma-1} \cos (2 \pi s) d s>0 .
$$

This shows that $c=\lim _{n \rightarrow \infty} n c_{n}$, and thus

$$
\lim _{n \rightarrow \infty} \int_{0}^{n c_{n}} s^{\gamma-1}(1-s / n)^{k} \cos (2 \pi s) d s=\int_{0}^{c} s^{\gamma-1} \cos (2 \pi s) d s>0 .
$$

Consequently, from (3.17) and (3.18), we see that

$$
\begin{aligned}
n a_{n} & >n \int_{0}^{c_{n}} f(s) \cos (2 \pi n s) d s \\
& =n^{1-\gamma} \int_{0}^{n c_{n}} s^{\gamma-1}(1-s / n)^{k} \cos (2 \pi s) d s \longrightarrow \infty
\end{aligned}
$$

as $n \rightarrow \infty$. This proves (3.11), and the proof is complete.

Proof of Theorem 3.5. Case 1. First we consider the semigroup case. As in Theorem 3.4, we take $(T(t))_{t \geq 0}$ to be the $C_{0}$-semigroup

$$
T(t):=e^{t(i a I+N)}=e^{i a t} \sum_{n=0}^{k} \frac{t^{n} N^{n}}{n !} \quad(t \geq 0),
$$

where $N$ is a bounded nilpotent operator on $X$ of order $k+1$ and $a \in \mathbb{R} \backslash\{0\}$. Since we have already observed that $\sup _{t>0}\left\|C_{t}^{k}\right\|<\infty$ (cf. (3.10)), it only remains to prove that $\sup _{t>0}\left\|C_{t}^{\gamma}\right\|=\infty$ for all $\gamma \in[0, k)$. 
Suppose $k-1<\gamma<k$. Applying (3.5) we have

$$
A C_{t}^{\gamma}=\frac{\gamma}{t}\left[C_{t}^{\gamma-1}-I\right]=\frac{\gamma}{t} C_{t}^{\gamma-1}+D_{t}^{1},
$$

where $\left\|D_{t}^{1}\right\|=\left\|t^{-1} \gamma I\right\|=O\left(t^{-1}\right)$ as $t \rightarrow \infty$; and

$$
A^{2} C_{t}^{\gamma}=\frac{\gamma(\gamma-1)}{t^{2}} C_{t}^{\gamma-2}+D_{t}^{2}
$$

where $\left\|D_{t}^{2}\right\|=O\left(t^{-1}\right)$ as $t \rightarrow \infty$. Continuing this process we find

$$
A^{k-1} C_{t}^{\gamma}=\frac{\gamma(\gamma-1) \ldots(\gamma-k+2)}{t^{k-1}} C_{t}^{\gamma-k+1}+D_{t}^{\gamma},
$$

where $\left\|D_{t}^{\gamma}\right\|=O\left(t^{-1}\right)$ as $t \rightarrow \infty$; and

$$
\begin{aligned}
C_{t}^{\gamma-k+1} & =\frac{\gamma-k+1}{t^{\gamma-k+1}} \int_{0}^{t}(t-s)^{\gamma-k} T(s) d s \\
& =\sum_{n=0}^{k} \frac{\gamma-k+1}{t^{\gamma-k+1} n !}\left(\int_{0}^{t}(t-s)^{\gamma-k} s^{n} e^{a i s} d s\right) N^{n} \\
& =\sum_{n=0}^{k} \frac{(\gamma-k+1) t^{n}}{n !}\left(\int_{0}^{1}(1-s)^{\gamma-k} s^{n} e^{a t i s} d s\right) N^{n}
\end{aligned}
$$

Hence

$$
\begin{aligned}
\frac{C_{t}^{\gamma-k+1}}{(\gamma-k+1) t^{k-1}}= & \sum_{n=0}^{k} \frac{t^{n-k+1}}{n !}\left(\int_{0}^{1}(1-s)^{\gamma-k} s^{n} e^{a t i s} d s\right) N^{n} \\
= & \frac{t}{k !}\left(\int_{0}^{1}(1-s)^{\gamma-k} s^{k} e^{a t i s} d s\right) N^{k} \\
& +\sum_{n=0}^{k-1} \frac{t^{n-k+1}}{n !}\left(\int_{0}^{1}(1-s)^{\gamma-k} s^{n} e^{a t i s} d s\right) N^{n} \\
= & B_{1}(t)+B_{2}(t),
\end{aligned}
$$

where $\left\|B_{2}(t)\right\|=o(1)$ as $t \rightarrow \infty$, because

$$
\lim _{t \rightarrow \infty} \int_{0}^{1}(1-s)^{\gamma-k} s^{n} e^{a t i s} d s=0
$$

for every $n \geq 0$ by the Riemann-Lebesgue theorem. On the other hand, since $-1<\gamma-k<0$, we can apply Lemma 3.6 to infer that $\lim _{\sup _{t \rightarrow \infty}}\left\|B_{1}(t)\right\|=\infty$.

Therefore we have $\lim \sup _{t \rightarrow \infty}\left\|t^{-(k-1)} C_{t}^{\gamma-k+1}\right\|=\infty$, and so

$$
\limsup _{t \rightarrow \infty}\left\|A^{k-1} C_{t}^{\gamma}\right\|=\infty
$$


by (3.21). This implies $\sup _{t>0}\left\|C_{t}^{\gamma}\right\|=\infty$. Hence, by Theorem 2.2(i), $\sup _{t>0}\left\|C_{t}^{\gamma}\right\|$ $=\infty$ for all $\gamma$ with $0 \leq \gamma<k$.

Case 2. Next we consider the cosine operator function case. Let $N: X \rightarrow X$ and $a \in \mathbb{R} \backslash\{0\}$ be the same as in Case 1. Then define $C(t): X \rightarrow X$ by

$$
\begin{aligned}
C(t) & =\frac{1}{2}\left\{e^{t(i a I+N)}+e^{-t(i a I+N)}\right\} \\
& =\sum_{n=0}^{k} \frac{1}{n !} \frac{\left(e^{i a t} t^{n}+e^{-i a t}(-t)^{n}\right)}{2} N^{n} \quad(t \geq 0) .
\end{aligned}
$$

Then, as is easily seen, $(C(t))_{t \geq 0}$ is a strongly continuous cosine operator function on $X$ with generator $B=(i a \bar{I}+N)^{2}$ (cf. Sova [12]). The Cesaro mean $C_{t}^{\gamma}$ of $(C(t))_{t \geq 0}$ of order $\gamma>0$ can be written as

$$
\begin{aligned}
C_{t}^{\gamma}= & \frac{\gamma}{t^{\gamma}} \int_{0}^{t}(t-s)^{\gamma-1} C(s) d s \\
= & \sum_{0 \leq n \leq k}^{\prime} \frac{\gamma t^{n}}{n !}\left(\int_{0}^{1}(1-s)^{\gamma-1} s^{n} \cos (a t s) d s\right) N^{n} \\
& +\sum_{0 \leq n \leq k}^{\prime \prime} \frac{\gamma t^{n}}{n !}\left(i \int_{0}^{1}(1-s)^{\gamma-1} s^{n} \sin (a t s) d s\right) N^{n}
\end{aligned}
$$

where $\sum_{0 \leq n \leq k}^{\prime}$ [resp. $\sum_{0 \leq n \leq k}^{\prime \prime}$ ] means that the summation is taken for all $n$ such that $0 \leq n \leq k$, and $n$ is even [resp. odd].

It is known (see e.g. [10], [12]) that

$$
C_{t}^{\alpha+2} B \subset B C_{t}^{\alpha+2}=\frac{(\alpha+2)(\alpha+1)}{t^{2}}\left[C_{t}^{\alpha}-I\right] \quad(t>0, \alpha \geq 0) ;
$$

and, since $w_{0}(C(\cdot))=0$,

$$
\lambda^{2}\left(\lambda^{2}-B\right)^{-1}=\lambda \int_{0}^{\infty} e^{-\lambda s} C(s) d s \quad(\operatorname{Re} \lambda>0) .
$$

For a moment we assume that $k=2 l$ for some integer $l \geq 1$. Using the above considerations we see as in Case 1 that

$$
B^{l} C_{t}^{k}=\frac{k !}{t^{2 l}} C(t)+E_{t}^{k}
$$

where $\left\|E_{t}^{k}\right\|=O\left(t^{-2}\right)$ as $t \rightarrow \infty$. Thus, by (3.23), $\sup _{t>0}\left\|B^{l} C_{t}^{k}\right\|<\infty$ and hence

$$
\sup _{t>0}\left\|C_{t}^{k}\right\| \leq \sup _{t>0}\left\|B^{-l}\right\|\left\|B^{l} C_{t}^{k}\right\|<\infty
$$


Next suppose $2 l-1<\gamma<2 l=k$. Then we see that

$$
B^{l-1} C_{t}^{\gamma}=\frac{\gamma(\gamma-1) \ldots(\gamma-2 l+3)}{t^{2 l-2}} C_{t}^{\gamma-2 l+2}+E_{t}^{\gamma},
$$

where $\left\|E_{t}^{\gamma}\right\|=O\left(t^{-2}\right)$ as $t \rightarrow \infty$. Write $\delta=\gamma-2 l+1$. Thus $0<\delta<1$, and

$$
\begin{aligned}
C_{t}^{\gamma-2 l+2}= & \sum_{0 \leq n \leq k}^{\prime} \frac{\gamma-2 l+2}{n !} t^{n}\left(\int_{0}^{1}(1-s)^{\delta} s^{n} \cos (a t s) d s\right) N^{n} \\
& +\sum_{0 \leq n \leq k}^{\prime \prime} \frac{\gamma-2 l+2}{n !} t^{n}\left(i \int_{0}^{1}(1-s)^{\delta} s^{n} \sin (a t s) d s\right) N^{n} .
\end{aligned}
$$

Now we apply integration by parts to get that

(i) if $n$ is an even integer such that $0 \leq n \leq 2 l=k$, then

$$
\begin{aligned}
& \int_{0}^{1}(1-s)^{\delta} s^{n} \cos (a t s) d s \\
& \quad=\frac{\delta}{a t} \int_{0}^{1}(1-s)^{\delta-1} s^{n} \sin (a t s) d s+\frac{1}{t^{2}} \cdot o(1) \quad(t \rightarrow \infty) ;
\end{aligned}
$$

(ii) if $n$ is an odd integer such that $0<n<2 l=k$, then

$$
\begin{aligned}
\int_{0}^{1} & (1-s)^{\delta} s^{n} \sin (a t s) d s \\
& =-\frac{\delta}{a t} \int_{0}^{1}(1-s)^{\delta-1} s^{n} \cos (a t s) d s+\frac{1}{t^{2}} \cdot o(1) \quad(t \rightarrow \infty) .
\end{aligned}
$$

Since

$$
\begin{aligned}
& \frac{1}{t^{2 l-2}} C_{t}^{\gamma-2 l+2}=\frac{1}{t^{2 l-2}}\left\{\sum_{0 \leq n \leq k}^{\prime} \frac{\gamma-2 l+2}{n !} t^{n}\left(\int_{0}^{1}(1-s)^{\delta} s^{n} \cos (\text { ats }) d s\right) N^{n}\right. \\
& \left.+\sum_{0 \leq n \leq k}^{\prime \prime} \frac{\gamma-2 l+2}{n !} t^{n}\left(i \int_{0}^{1}(1-s)^{\delta} s^{n} \sin (a t s) d s\right) N^{n}\right\} \\
& =I_{1}(t)+I_{2}(t) \text {, }
\end{aligned}
$$

where

$$
I_{1}(t):=\frac{\gamma-2 l+2}{(2 l) !} t^{2}\left(\int_{0}^{1}(1-s)^{\delta} s^{2 l} \cos (a t s) d s\right) N^{2 l}
$$

and

$$
I_{2}(t):=\frac{1}{t^{2 l-2}} C_{t}^{\gamma-2 l+2}-I_{1}(t)
$$


we obtain, by applying (3.30), (3.31) and Lemma 3.6, that $\lim \sup _{t \rightarrow \infty}\left\|I_{1}(t)\right\|=$ $\infty$, and that $\lim _{t \rightarrow \infty}\left\|I_{2}(t)\right\|=0$. Hence it follows from (3.29) that $\sup _{t>0}\left\|C_{t}^{\gamma}\right\|$ $=\infty$. Since this holds for any $\gamma$ with $2 l-1<\gamma<2 l=k$, it follows from Theorem 2.2(i) that $\sup _{t>0}\left\|C_{t}^{\gamma}\right\|=\infty$ for all $\gamma$ with $0 \leq \gamma<k$.

Assume next that $k=2 l+1$ for some integer $l \geq 0$. Then as before we have

$$
B^{l} C_{t}^{k}=\frac{k !}{t^{2 l}} C_{t}^{1}+F_{t}^{k}
$$

where $\left\|F_{t}^{k}\right\|=O\left(t^{-2}\right)$ as $t \rightarrow \infty$, and

$$
C_{t}^{1}=\sum_{0 \leq n \leq k}^{\prime} \frac{t^{n}}{n !}\left(\int_{0}^{1} s^{n} \cos (\text { ats }) d s\right) N^{n}+\sum_{0 \leq n \leq k}^{\prime \prime} \frac{t^{n}}{n !}\left(i \int_{0}^{1} s^{n} \sin (\text { ats }) d s\right) N^{n}
$$

We have:

(i) if $n$ is an even integer such that $0 \leq n<2 l+1=k$, then

$$
\int_{0}^{1} s^{n} \cos (a t s) d s=\frac{\sin a t}{a t}+o\left(t^{-1}\right) \quad(t \rightarrow \infty) ;
$$

(ii) if $n$ is an odd integer such that $0<n \leq 2 l+1=k$, then

$$
\int_{0}^{1} s^{n} \sin (a t s) d s=\frac{\cos a t}{-a t}+o\left(t^{-1}\right) \quad(t \rightarrow \infty) .
$$

Thus $\left\|B^{l} C_{t}^{k}\right\|=O(1)$ as $t \rightarrow \infty$, and hence as before

$$
\sup _{t>0}\left\|C_{t}^{k}\right\| \leq \sup _{t>0}\left\|B^{-l}\right\| \cdot\left\|B^{l} C_{t}^{k}\right\|<\infty .
$$

Finally, let $\gamma>0$ be such that $2 l<\gamma<2 l+1=k$. Then

$$
B^{l} C_{t}^{\gamma}=\frac{\gamma(\gamma-1) \ldots(\gamma-2 l+1)}{t^{2 l}} C_{t}^{\gamma-2 l}+F_{t}^{\gamma},
$$

where $\left\|F_{t}^{\gamma}\right\|=O\left(t^{-2}\right)$ as $t \rightarrow \infty$. Write $\delta=\gamma-2 l$. Thus $0<\delta<1$, and

$$
\begin{aligned}
C_{t}^{\gamma-2 l}= & \sum_{0 \leq n \leq k}^{\prime} \frac{\gamma-2 l}{n !} t^{n}\left(\int_{0}^{1}(1-s)^{\delta-1} s^{n} \cos (\text { ats }) d s\right) N^{n} \\
& +\sum_{0 \leq n \leq k}^{\prime \prime} \frac{\gamma-2 l}{n !} t^{n}\left(i \int_{0}^{1}(1-s)^{\delta-1} s^{n} \sin (\text { ats }) d s\right) N^{n} .
\end{aligned}
$$

From Lemma 3.6 and (3.33) we see that $\limsup _{t \rightarrow \infty}\left\|B^{l} C_{t}^{\gamma}\right\|=\infty$. Therefore, $\sup _{t>0}\left\|C_{t}^{\gamma}\right\|=\infty$. Since this holds for any $\gamma$ with $2 l<\gamma<2 l+1=k$, it follows as before that $\sup _{t>0}\left\|C_{t}^{\gamma}\right\|=\infty$ for all $\gamma$ with $0 \leq \gamma<2 l+1=k$. 
This completes the proof of Theorem 3.5.

Remark. Let $(C(t))_{t \geq 0}$ be a strongly continuous cosine operator function on $X$ with generator $B$. If $X \neq\{0\}$, then $0 \leq \sigma(C(\cdot)) \leq w_{0}(C(\cdot))<\infty$, where $\sigma(C(\cdot))$ is defined analogously to $\sigma(T(\cdot))$ (cf. (3.1)). To see this we first note that $w_{0}(C(\cdot))<\infty$ by [12], and $\sigma(C(\cdot)) \leq w_{0}(C(\cdot))$ is obvious. Thus it suffices to prove that $\sigma(C(\cdot)) \geq 0$. Suppose $K:=\sigma(C(\cdot))<0$. Then an elementary calculation (see e.g. [12, pp. 24-25]) shows that if $\lambda \neq 0$ satisfies $\operatorname{Re} \lambda>K$, then $\lambda^{2} \in \rho(B)$ and $\int_{0}^{\infty} e^{-\lambda t} C(t) d t=\lambda\left(\lambda^{2}-B\right)^{-1}$. On the other hand, since the operator-valued function $\lambda \mapsto \int_{0}^{\infty} e^{-\lambda t} C(t) d t$ (defined strongly) is analytic on the domain $\Omega:=\{\lambda: \operatorname{Re} \lambda>K\}$ (cf. the proof of Theorem 1.5.1 of [1]), it follows that

$$
\lim _{|\lambda| \rightarrow 0}\left\|\int_{0}^{\infty} e^{-\lambda t} C(t) d t\right\|=\left\|\int_{0}^{\infty} C(t) d t\right\|<\infty .
$$

Hence

$$
\lim _{|\lambda| \rightarrow 0}\left\|\lambda(\lambda-B)^{-1}\right\|=\lim _{|\lambda| \rightarrow 0}\left\|\lambda \int_{0}^{\infty} e^{-\lambda t} C(t) d t\right\|=0
$$

and thus, $0 \in \rho(B)$ by Theorem 3.1 of [11]. Then, since $\lim _{|\lambda| \rightarrow 0}\left\|\lambda\left(\lambda^{2}-B\right)^{-1}\right\|=$ 0 , we have

$$
\left\|\int_{0}^{\infty} C(t) d t\right\|=\lim _{|\lambda| \rightarrow 0}\left\|\int_{0}^{\infty} e^{-\lambda t} C(t) d t\right\|=\lim _{|\lambda| \rightarrow 0}\left\|\lambda\left(\lambda^{2}-B\right)^{-1}\right\|=0,
$$

which implies $\int_{0}^{\infty} C(t) x d t=0$ for all $x \in X$, i.e., $\lim _{t \rightarrow \infty} \int_{0}^{t} C(s) x d s=0$ for all $x \in X$. Thus, by Theorem 2.3 of [11], we must have $X=\{0\}$, a contradiction.

The above proof of Theorem 3.5 turns up the following result.

Theorem 3.7. There exists a $C_{0}$-semigroup $(T(t))_{t \geq 0}$ [resp. a strongly continuous cosine operator function $(C(t))_{t \geq 0}$ l of bounded linear operators on $X$ such that $\sup _{t>0}\left\|C_{t}^{\gamma}\right\|=\infty$ for all $\gamma>0$, but $\sup _{\lambda>0}\left\|\lambda(\lambda-A)^{-1}\right\|<\infty$ [resp. $\sup _{\lambda>0}\left\|\lambda(\lambda-B)^{-1}\right\|<\infty$ ], where $A$ [resp. B] denotes the generator of $(T(t))_{t \geq 0}\left[\right.$ resp. $\left.(C(t))_{t \geq 0}\right]$.

Proof. Fix $a \in \mathbb{R}$ with $|a|>2$. It follows from the proof of Theorem 3.5 that, for each $k \geq 1$, letting $N_{k}: X_{k} \rightarrow X_{k}$ be a bounded linear operator on a Banach space $X_{k}$ such that $N_{k}^{k} \neq 0, N_{k}^{k+1}=0$, and $\left\|N_{k}\right\|_{X_{k}}<1$; and defining

$$
T_{k}(t):=e^{t\left(i a I+N_{k}\right)}=e^{i a t} \sum_{n=0}^{k} \frac{t^{n}}{n !} N_{k}^{n} \quad(t \geq 0),
$$


we have a $C_{0}$-semigroup $\left(T_{k}(t)\right)_{t \geq 0}$ on $X_{k}$, with generator $A_{k}=i a+N_{k}$, such that

$$
\sup _{t>0}\left\|C_{t}^{k-1}\left(T_{k}(\cdot)\right)\right\|_{X_{k}}=\infty, \quad \text { and } \quad \sup _{t>0}\left\|C_{t}^{k}\left(T_{k}(\cdot)\right)\right\|_{X_{k}}<\infty .
$$

Furthermore, since $\left\|N_{k}\right\|_{X_{k}}<1$ and $|a|>2$, and since

$$
A_{k}^{-1}=\left(i a I+N_{k}\right)^{-1}=\frac{1}{i a}\left(I+\frac{N_{k}}{i a}\right)^{-1}=\frac{1}{i a} \sum_{n=0}^{k}\left(\frac{-1}{i a}\right)^{n} N_{k}^{n},
$$

it follows that

$$
\left\|A_{k}^{-1}\right\|_{X_{k}} \leq \frac{1}{|a|} \sum_{n=0}^{k}\left(\frac{1}{|a|}\right)^{n}<1 .
$$

On the other hand, as in (3.21), we have

$$
\left(A_{k}\right)^{k} C_{t}^{k}\left(T_{k}(\cdot)\right)=\frac{k !}{t^{k}} C_{t}^{0}\left(T_{k}(\cdot)\right)+D_{t}^{k}=e^{i a t} \sum_{n=0}^{k} \frac{k !}{n !} t^{n-k} N_{k}^{n}+D_{t}^{k},
$$

where $\left\|D_{t}^{k}\right\|_{X_{k}}=O\left(t^{-1}\right)$ as $t \rightarrow \infty$. Thus there exists $G_{k} \in \mathbb{R}$, with $G_{k}>(k+1)$ !, such that $t>G_{k}$ implies $\left\|D_{t}^{k}\right\|_{X_{k}}<1$. Then by (3.36)

$$
\left\|C_{t}^{k}\left(T_{k}(\cdot)\right)\right\|_{X_{k}} \leq\left\|\sum_{n=0}^{k} \frac{k !}{n !} t^{n-k} N_{k}^{n}\right\|_{X_{k}}+1 \quad \text { for all } t>G_{k} .
$$

If we choose $N_{k}$ in such a way that $\left\|N_{k}\right\|_{X_{k}}$ is sufficiently small (in particular, $\left.\left\|N_{k}\right\|_{X_{k}}<\ln 2\right)$ and $\lim _{n \rightarrow \infty}\left\|N_{k}\right\|_{X_{k}}=0$, then we can assume that

$$
\left\|T_{k}(t)\right\|_{X_{k}}=\left\|e^{i a t} \sum_{n=0}^{k} \frac{t^{n}}{n !} N_{k}^{n}\right\|_{X_{k}}<2 \quad\left(0 \leq t \leq G_{k}\right),
$$

and

$$
\left\|\sum_{n=0}^{k} \frac{k !}{n !} t^{n-k} N_{k}^{n}\right\|_{X_{k}} \leq 1 \quad\left(t>G_{k}>(k+1) !\right) .
$$

Hence it follows from (3.37) that

$$
\sup _{t>0}\left\|C_{t}^{k}\left(T_{k}(\cdot)\right)\right\|_{X_{k}} \leq 2,
$$


so that by Theorem 2.7(ii)

$$
\sup _{\lambda>0}\left\|\lambda\left(\lambda-A_{k}\right)^{-1}\right\|_{X_{k}}=\sup _{\lambda>0}\left\|\lambda \int_{0}^{\infty} e^{\lambda s} T_{k}(s) d s\right\| \leq 2 .
$$

Now, define

$$
X:=\left\{x=\left(x_{1}, x_{2}, \ldots\right): x_{k} \in X_{k}, \sum_{k=1}^{\infty}\left\|x_{k}\right\|<\infty\right\}, \quad \text { and } \quad\|x\|:=\sum_{k=1}^{\infty}\left\|x_{k}\right\|
$$

for $x \in X$. Then $X$ becomes a Banach space. If $t \geq 0$, define a bounded linear operator $T(t)$ on $X$ by

$$
T(t) x:=\left(T_{1}(t) x_{1}, T_{2}(t) x_{2}, \ldots\right) \quad \text { for } \quad x=\left(x_{1}, x_{2}, \ldots\right) \in X .
$$

By (3.38) we see that $\|T(t)\|<2$ for all $0 \leq t \leq 1$, and hence $\lim _{t \downarrow 0}\|T(t) x-x\|=$ 0 for all $x \in X$. Therefore, $(T(t))_{t \geq 0}$ is a $C_{0}$-semigroup of bounded linear operators on $X$. Since $\left\|N_{k}\right\|_{X_{k}}<\ln 2$, it follows that

$$
\left\|\sum_{n=0}^{k} \frac{t^{n}}{n !} N_{k}^{n}-I\right\|_{X_{k}} \leq \sum_{n=1}^{k} \frac{t^{n}}{n !}\left\|N_{k}\right\|_{X_{k}}^{n}<e^{t\left\|N_{k}\right\|_{X_{k}}-1<2^{t}-1}
$$

for all $t>0$. Thus by (3.34)

$$
\left\|T_{k}(t)-I\right\|_{X_{k}} \leq\left\|\sum_{n=0}^{k} \frac{t^{n}}{n !} N_{k}^{n}-I\right\|_{X_{k}}+\left|e^{i a t}-1\right|<\left(2^{t}-1\right)+\left|e^{i a t}-1\right|
$$

for all $t>0$. Hence

$$
\lim _{t \downarrow 0}\|T(t)-I\| \leq \lim _{t \downarrow 0}\left(\sup _{k \geq 1}\left\|T_{k}(t)-I\right\|_{X_{k}}\right)=0 .
$$

It follows that $(T(t))_{t \geq 0}$ has generator $A$ on $X$, where $A$ is defined by

$$
A x=A\left(x_{1}, x_{2}, \ldots\right):=\left(A_{1} x_{1}, A_{2} x_{2}, \ldots\right) \text { for } \quad x=\left(x_{1}, x_{2}, \ldots\right) \in X .
$$

Clearly, $A$ is a bounded linear operator on $X$ with $\|A\| \leq \sup _{k \geq 1}\left\|A_{k}\right\|_{X_{k}}=$ $\sup _{k \geq 1}\left\|i a+N_{k}\right\|_{X_{k}} \leq|a|+1$.

Next, suppose $\lambda>0$. Since $\sigma(T(\cdot))=w_{0}(T(\cdot))=0$ by the definition of $(T(t))_{t \geq 0}$, we see that $\lambda \in \rho(A)$; and furthermore

$$
(\lambda-A)^{-1} x=(\lambda-A)^{-1}\left(x_{1}, x_{2}, \ldots\right)=\left(\left(\lambda-A_{1}\right)^{-1} x_{1},\left(\lambda-A_{2}\right)^{-1} x_{2}, \ldots\right)
$$


for $x=\left(x_{1}, x_{2}, \ldots\right) \in X$. Consequently

$$
\begin{aligned}
\sup _{\lambda>0}\left\|\lambda(\lambda-A)^{-1}\right\| & =\sup _{\lambda>0}\left\|\lambda \int_{0}^{\infty} e^{-\lambda s} T(s) d s\right\| \\
& \leq \sup _{\lambda>0}\left(\sup _{n \geq 1}\left\|\lambda\left(\lambda-A_{k}\right)^{-1}\right\|_{X_{k}}\right) \leq 2 .
\end{aligned}
$$

On the other hand, if $\gamma>0$, then for all integers $k$ with $k>\gamma+1$ we have, by Theorem 2.2(i) and (3.35),

$$
\sup _{t>0}\left\|C_{t}^{\gamma}\right\| \geq \sup _{t>0}\left\|C_{t}^{k-1}\right\| \geq \sup _{t>0}\left\|C_{t}^{k-1}\left(T_{k}(\cdot)\right)\right\|_{X_{k}}=\infty .
$$

This completes the proof for the semigroup case.

The proof for the cosine operator function case is an easy modification of the above proof, since the cosine operator function $(C(t))_{t \geq 0}$ in Theorem 3.5 was defined by (3.23). Hence we may omit the details here.

\section{Theorem 2.9 Cannot be Extended to the Case $0 \leq \gamma<1$}

We first state a consequence of Theorem 2.9 and Corollary 2.10 as follows.

Theorem 4.1. (Cf. [8, Corollary 3.2].) Let $(T(t))_{t \geq 0}$ [resp. $\left.(C(t))_{t \geq 0}\right]$ be a $C_{0}$-semigroup [resp. a strongly continuous cosine operator function] of positive linear operators on a Banach lattice $X$ with generator $A$ [resp. B]. Then the following hold.

(i) If $\gamma \geq 1$ and $\alpha>-1-\gamma$, then the boundedness of $\left\{t^{-\alpha} C_{t}^{\gamma}: t>0\right\}$ is equivalent to $\sigma(T(\cdot)) \leq 0$ [resp. $\sigma(C(\cdot))=0$ ] together with the boundedness of $\left\{\lambda^{\alpha+1}(\lambda-A)^{-1}: \lambda>0\right\}$ [resp. $\left\{\lambda^{\alpha+2}\left(\lambda^{2}-B\right)^{-1}: \lambda>0\right\}$ ]. In particular, $(T(t))_{t \geq 0}$ [resp. $(C(t))_{t \geq 0}$ ] is Abel-mean-bounded if and only if it is $\gamma$-Cesaro-mean-bounded for any $\gamma \geq 1$.

(ii) For all $\gamma \geq 1$ and $\alpha>-2,\left\|C_{t}^{\gamma}\right\|=O\left(t^{\alpha}\right)$ as $t \rightarrow \infty$ is equivalent to $\sigma(T(\cdot)) \leq 0$ [resp. $\sigma(C(\cdot))=0$ ] together with $\left\|\lambda(\lambda-A)^{-1}\right\|=O\left(\lambda^{-\alpha}\right)$ [resp. $\left.\left\|\lambda^{2}\left(\lambda^{2}-B\right)^{-1}\right\|=O\left(\lambda^{-\alpha}\right)\right]$ as $\lambda \downarrow 0$.

(iii) If $X \neq\{0\}$ and $\sigma(T(\cdot)) \leq 0$ [resp. $\sigma(C(\cdot))=0$ ], then $\alpha_{0}\left(C^{\gamma}\right)=\alpha_{0}(A$. $) \geq$ -1 for all $\gamma \geq 1$, where the Abel mean $A_{\lambda}=\lambda \int_{0}^{\infty} e^{-\lambda t} T(t) d t$ [resp. $\left.A_{\lambda}=\lambda \int_{0}^{\infty} e^{-\lambda t} C(t) d t\right]$ is defined strongly for all $\lambda>0$.

In the next two theorems, we present examples to show that the equivalence of the Abel-mean-boundedness and the $\gamma$-Cesarro-mean-boundedness is not true for the case $0 \leq \gamma<1$. 
Theorem 4.2. Let $0<\gamma<1$. Then there exists a $C_{0}$-semigroup $(T(t))_{t \geq 0}$ [resp. a strongly continuous cosine operator function $(C(t))_{t \geq 0}$ ] of positive linear operators on an $L_{1}$-space such that $\sup _{t>0}\left\|C_{t}^{\gamma}\right\|_{1}=\infty$, but $\sup _{t>0}\left\|C_{t}^{\beta}\right\|_{1}<\infty$ for all $\beta>\gamma$ and, in particular, the Abel means of $(T(t))_{t \geq 0}\left[\right.$ resp. $\left.(C(t))_{t \geq 0}\right]$ are uniformly bounded.

Lemma 4.3. (a) Let $\xi_{j}, \eta_{j}>0, \sum_{j=1}^{n} \xi_{j}=1=\sum_{j=1}^{n} \eta_{j}$, and $\xi_{j} / \xi_{j+1} \geq$ $\eta_{j} / \eta_{j+1}(1 \leq j \leq n-1)$. Then $\lambda_{1} \geq \lambda_{2} \geq \ldots \geq \lambda_{n}$ implies

$$
\sum_{j=1}^{n} \lambda_{j} \xi_{j} \geq \sum_{j=1}^{n} \lambda_{j} \eta_{j}
$$

(b) Let $a_{1} \geq a_{2} \geq \ldots \geq a_{n}$, and $b_{1} \geq b_{2} \geq \ldots \geq b_{n}$. Then

$$
\sum_{j=1}^{n} a_{j} b_{j} \geq \sum_{j=1}^{n} a_{j} b_{k(j)}
$$

where $\{k(j) ; 1 \leq j \leq n\}=\{1,2, \ldots, n\}$.

Proof. We prove (a) by induction. The case $n=2$ is obvious. Next, suppose that the case $k=n$ is true, and we consider the case $k=n+1$. Let $\xi_{j}, \eta_{j}>0(j=1,2, \ldots, n+1)$ be such that $\sum_{j=1}^{n+1} \xi_{j}=1=\sum_{j=1}^{n+1} \eta_{j}$ and $\xi_{j} / \xi_{j+1} \geq \eta_{j} / \eta_{j+1}(1 \leq j \leq n)$. Clearly, $\xi_{n-1} / \xi_{n+1} \geq \eta_{n-1} / \eta_{n+1}$, and hence $\xi_{n-1} \eta_{n+1} \geq \eta_{n-1} \xi_{n+1}$; similarly $\xi_{n-1} \eta_{n} \geq \eta_{n-1} \xi_{n}$. Thus

$$
\begin{gathered}
\xi_{n-1}\left(\eta_{n}+\eta_{n+1}\right)-\eta_{n-1}\left(\xi_{n}+\xi_{n+1}\right)=\xi_{n-1} \eta_{n}+\xi_{n-1} \eta_{n+1}-\eta_{n-1} \xi_{n}-\eta_{n-1} \xi_{n+1} \\
=\left(\xi_{n-1} \eta_{n+1}-\eta_{n-1} \xi_{n+1}\right)+\left(\xi_{n-1} \eta_{n}-\eta_{n-1} \xi_{n}\right) \geq 0,
\end{gathered}
$$

which implies $\xi_{n-1} /\left(\xi_{n}+\xi_{n+1}\right) \geq \eta_{n-1} /\left(\eta_{n}+\eta_{n+1}\right)$. Hence we can apply the induction hypothesis: If $\lambda_{1} \geq \lambda_{2} \geq \ldots \geq \lambda_{n} \geq \lambda_{n+1}$, then

$$
\sum_{j=1}^{n-1} \lambda_{j} \xi_{j}+\lambda_{n}\left(\xi_{n}+\xi_{n+1}\right) \geq \sum_{j=1}^{n-1} \lambda_{j} \eta_{j}+\lambda_{n}\left(\eta_{n}+\eta_{n+1}\right) .
$$

On the other hand, by the assumptions on $\xi_{j}, \eta_{j}(j=1,2, \ldots, n+1)$, it follows at once that $\xi_{n+1} \leq \eta_{n+1}$. Since $\lambda_{n+1}-\lambda_{n} \leq 0$, it follows that $\left(\lambda_{n+1}-\lambda_{n}\right) \xi_{n+1} \geq$ $\left(\lambda_{n+1}-\lambda_{n}\right) \eta_{n+1}$. Therefore

$$
\begin{aligned}
\sum_{j=1}^{n+1} \lambda_{j} \xi_{j} & =\sum_{j=1}^{n-1} \lambda_{j} \xi_{j}+\lambda_{n}\left(\xi_{n}+\xi_{n+1}\right)+\left(\lambda_{n+1}-\lambda_{n}\right) \xi_{n+1} \\
& \geq \sum_{j=1}^{n-1} \lambda_{j} \eta_{j}+\lambda_{n}\left(\eta_{n}+\eta_{n+1}\right)+\left(\lambda_{n+1}-\lambda_{n}\right) \eta_{n+1}=\sum_{j=1}^{n+1} \lambda_{j} \eta_{j},
\end{aligned}
$$


completing the proof of (a).

(b) is a part of the well-known rearrangement inequality and follows from an easy induction argument. We may omit the proof.

Proof of Theorem 4.2. This is an adaptation of the argument of [8, Proposition 4.3]. Let $a>2$ be the unique solution of the equation $2=a^{\gamma}$. For $j \geq 1$, let (4.1)

$$
X_{j}:=\left[0, a^{j}\right) \text { and } w_{j}(s):= \begin{cases}2^{j} & \left(0 \leq s<a^{0}=1\right), \\ 2^{j-1-k} & \left(a^{k} \leq s<a^{k+1}, 0 \leq k \leq j-1\right) .\end{cases}
$$

For $t \geq 0$, let $\vartheta_{j, t}: X_{j} \rightarrow X_{j}$ be the point transformation defined by $\vartheta_{j, t}(s):=s+t$ $\left(\bmod a^{j}\right)$, and then put $T_{j}(t) f(s):=f\left(\vartheta_{j, t}(s)\right)$ for $f$ on $X_{j}$, and define a measure $\mu_{j}$ on $X_{j}$ by $\mu_{j}:=w_{j}(s) d s$.

Let

$$
X:=\bigcup_{j=1}^{\infty} X_{j} \quad \text { (regarded as a disjoint union), }
$$

and let $\mu$ be the measure on $X$ defined by $\left.\mu\right|_{X_{j}}:=\mu_{j}$ for each $j \geq 1$. For $t \geq 0$, define an operator $T(t): L_{1}(X, \mu) \rightarrow L_{1}(X, \mu)$ by $\left.(T(t) f)\right|_{X_{j}}:=T_{j}(t)\left(\left.f\right|_{X_{j}}\right)$ for $j \geq 1$. It follows that $(T(t))_{t \geq 0}$ becomes a $C_{0}$-semigroup of positive linear operators on $L_{1}(X, \mu)$.

We will prove that $\sup _{t>0}\left\|C_{t}^{\gamma}\right\|_{1}=\infty$, and that $\sup _{t>0}\left\|C_{t}^{\beta}\right\|_{1}<\infty$ for all $\beta>\gamma$. To do this, let $0<\alpha<1$ be any real number. We consider the Cesaro means $C_{t}^{\alpha}$ of $(T(t))_{t \geq 0}$ of order $\alpha$, and define

$$
\alpha\left(X_{j}\right):=\sup \left\{\left\|C_{t}^{\alpha} f\right\|_{1} /\|f\|_{1}: 0 \neq f \in L_{1}\left(X_{j}, \mu_{j}\right) ; t>0\right\} .
$$

Then it suffices to show that $\sup _{j \geq 1} \alpha\left(X_{j}\right)<\infty$ for $\alpha=\beta>\gamma$, and $\lim _{j \rightarrow \infty} \alpha\left(X_{j}\right)=$ $\infty$ for $0<\alpha \leq \gamma$.

(i) To estimate $\alpha\left(X_{j}\right)$, let $f_{\delta}:=\delta^{-1} \chi_{\left[a^{j}-\delta, a^{j}\right)}$ for $0<\delta<1$. We have $\left\|f_{\delta}\right\|_{1}=1$, and

$$
\left\|C_{a^{j}}^{\alpha} f_{\delta}\right\|_{1} \leq \frac{\alpha}{a^{j \alpha}} \int_{0}^{a^{j}} t^{\alpha-1}\left\|T\left(a^{j}-t\right) f_{\delta}\right\|_{1} d t
$$

Denoting

$$
D_{j}:=\int_{a^{j-1}}^{a^{j}} t^{\alpha-1} 2^{0} d t+\int_{a^{j-2}}^{a^{j-1}} t^{\alpha-1} 2^{1} d t+\ldots+\int_{0}^{1} t^{\alpha-1} 2^{j} d t
$$

and noting that $w_{j}$ is non-increasing on $\left[0, a^{j}\right)$, we easily see that

$$
D_{j}-\int_{0}^{1} t^{\alpha-1} 2^{j} d t<\int_{0}^{a^{j}} t^{\alpha-1}\left\|T\left(a^{j}-t\right) f_{\delta}\right\|_{1} d t<2 D_{j} .
$$


Since

$$
\begin{aligned}
D_{j}= & \frac{1}{\alpha}\left[2^{0}\left(a^{j \alpha}-a^{(j-1) \alpha}\right)+2\left(a^{(j-1) \alpha}-a^{(j-2) \alpha}\right)+\ldots\right. \\
& \left.+2^{j-1}\left(a^{\alpha}-a^{0}\right)+2^{j} a^{0}\right] \\
= & \frac{1-a^{-\alpha}}{\alpha}\left[2^{0} a^{j \alpha}+2^{1} a^{(j-1) \alpha}+\ldots+2^{j-1} a^{\alpha}+2^{j} a^{0}\right]+\frac{1}{\alpha} 2^{j} a^{-\alpha} \\
= & \frac{\left(1-a^{-\alpha}\right) a^{j \alpha}}{\alpha} \sum_{k=0}^{j}\left(\frac{2}{a^{\alpha}}\right)^{k}+\frac{a^{j \alpha}}{\alpha}\left(\frac{2}{a^{\alpha}}\right)^{j} \frac{1}{a^{\alpha}}
\end{aligned}
$$

and

$$
D_{j}-\int_{0}^{1} t^{\alpha-1} 2^{j} d t=\frac{\left(1-a^{-\alpha}\right) a^{j \alpha}}{\alpha} \sum_{k=0}^{j-1}\left(\frac{2}{a^{\alpha}}\right)^{k},
$$

it follows that

$$
\left\|C_{a^{j}}^{\alpha} f_{\delta}\right\|_{1}>\left(1-a^{-\alpha}\right) \sum_{k=0}^{j-1}\left(\frac{2}{a^{\alpha}}\right)^{k}
$$

and

$$
\begin{aligned}
\left\|C_{a^{j}}^{\alpha} f_{\delta}\right\|_{1}<\frac{\alpha}{a^{j \alpha}} 2 D_{j} & =2\left\{\left(1-a^{-\alpha}\right) \sum_{k=0}^{j}\left(\frac{2}{a^{\alpha}}\right)^{k}+\left(\frac{2}{a^{\alpha}}\right)^{j} \frac{1}{a^{\alpha}}\right\} \\
& <2 \sum_{k=0}^{j}\left(\frac{2}{a^{\alpha}}\right)^{k} .
\end{aligned}
$$

(ii) Next, let $g_{d}:=\delta^{-1} \chi_{[d-\delta, d)}$, where $0<\delta<1$ and $a^{j-1} \leq d-\delta<d \leq a^{j}$. Then $\left\|g_{d}\right\|_{1}=1$, and

$$
\begin{aligned}
\left\|C_{d}^{\alpha} g_{d}\right\|_{1} & =\frac{\alpha}{d^{\alpha}} \int_{0}^{d} t^{\alpha-1}\left\|T(d-t) g_{d}\right\|_{1} d t \\
& =\frac{\alpha}{d^{\alpha}}\left(\int_{a^{j-1}}^{d}+\int_{0}^{a^{j-1}}\right) t^{\alpha-1}\left\|T(d-t) g_{d}\right\|_{1} d t \\
& =I+I I .
\end{aligned}
$$

It follows immediately that

$$
I<\frac{\alpha}{d^{\alpha}} \int_{a^{j-1}}^{d} t^{\alpha-1} \cdot 2 d t<2 .
$$


Letting $h_{d}:=T\left(d-a^{j-1}\right) g_{d}$, we see that $\left.h_{d}=\delta^{-1} \chi_{\left[a^{j-1}-\delta, a^{j-1}\right.}\right)$ and hence $\left\|h_{d}\right\|_{1}=2$. Then, since $T(d-t) g_{d}=T\left(\left(d-a^{j-1}\right)+a^{j-1}-t\right) g_{d}=T\left(a^{j-1}-\right.$ t) $T\left(d-a^{j-1}\right) g_{d}=T\left(a^{j-1}-t\right) h_{d}$, we obtain, as in (i), that

$$
\begin{aligned}
I I & =\frac{\alpha}{d^{\alpha}} \int_{0}^{a^{j-1}} t^{\alpha-1}\left\|T\left(a^{j-1}-t\right) h_{d}\right\|_{1} d t<\left\|C_{a^{j-1}}^{\alpha} h_{d}\right\|_{1} \\
& \leq \frac{\alpha}{a^{(j-1) \alpha}}\left(\int_{1}^{a^{j-1}} t^{\alpha-1}\left\|T\left(a^{j-1}-t\right) h_{d}\right\|_{1} d t+\int_{0}^{1} t^{\alpha-1} 2 \cdot 2^{j-1} d t\right) \\
& <\frac{\alpha}{a^{(j-1) \alpha}} 4 D_{j-1}<4 \sum_{k=0}^{j-1}\left(\frac{2}{a^{\alpha}}\right)^{k} \quad \text { (cf. (4.7)). }
\end{aligned}
$$

\section{Consequently}

$$
\left\|C_{d}^{\alpha} g_{d}\right\|_{1}=I+I I<2+4 \sum_{k=0}^{j-1}\left(\frac{2}{a^{\alpha}}\right)^{k} .
$$

(iii) Assume that $0<r<d$. Since $\left\|T(t) g_{d}\right\|_{1} \leq 2$ for all $0 \leq t \leq 1$, it follows that if $0<r \leq 1$, then

$$
\left\|C_{r}^{\alpha} g_{d}\right\|_{1}=\frac{\alpha}{r^{\alpha}} \int_{0}^{r} t^{\alpha-1}\left\|T(r-t) g_{d}\right\|_{1} d t \leq 2 .
$$

Next, if $1<r<d$, then choose an integer $j^{\prime}$, with $1 \leq j^{\prime} \leq j$, such that $a^{j^{\prime}-1}<r \leq a^{j^{\prime}}$. Let $0<\delta^{\prime}<1$ be such that $a^{j^{\prime}-1} \leq r-\delta^{\prime}<r \leq a^{j^{\prime}}$, and define

$$
g_{r}^{\prime}:=\frac{1}{2^{\left(j-j^{\prime}\right)} \delta^{\prime}} \chi_{\left[r-\delta^{\prime}, r\right)} .
$$

It follows that $\left\|g_{r}^{\prime}\right\|_{1}=1$, and that

$$
\left\|T(r-t) g_{d}\right\|_{1} \leq \begin{cases}2\left\|T(r-t) g_{r}^{\prime}\right\|_{1} & (1 \leq t \leq r), \\ 2^{j^{\prime}} & (0 \leq t \leq 1) .\end{cases}
$$

Hence as in (ii)

$$
\begin{aligned}
& \left\|C_{r}^{\alpha} g_{d}\right\|_{1} \\
& \quad<\frac{\alpha}{r^{\alpha}}\left(2 \int_{1}^{r} t^{\alpha-1}\left\|T(r-t) g_{r}^{\prime}\right\|_{1} d t+2 \int_{0}^{1} t^{\alpha-1} 2^{j^{\prime}} d t\right) \\
& \quad<2\left(2+\frac{\alpha}{a^{\left(j^{\prime}-1\right) \alpha}}\left(\int_{1}^{a^{j^{\prime}-1}} t^{\alpha-1}\left\|T(r-t) g_{r}^{\prime}\right\|_{1} d t+\int_{0}^{1} t^{\alpha-1} 2 \cdot 2^{j^{\prime}-1} d t\right)\right) \\
& \quad<2\left(2+\frac{\alpha}{a^{\left(j^{\prime}-1\right) \alpha}} 4 D_{j^{\prime}-1}\right)<2\left(2+4 \sum_{k=0}^{j^{\prime}-1}\left(\frac{2}{a^{\alpha}}\right)^{k}\right)
\end{aligned}
$$




$$
\leq 2\left(2+4 \sum_{k=0}^{j-1}\left(\frac{2}{a^{\alpha}}\right)^{k}\right) .
$$

This holds in particular for the case $0<r<d=a^{j}$ and $g_{a^{j}}=f_{\delta}$.

(iv) Now we estimate $\left\|C_{r}^{\alpha} f_{\delta}\right\|_{1}$ for $r>a^{j}$. To do this, choose an integer $n \geq 1$ such that $n a^{j}<r \leq(n+1) a^{j}$. Then we have

$$
\left\|C_{r}^{\alpha} f_{\delta}\right\|_{1}=\frac{\alpha}{r^{\alpha}}\left(\int_{r-n a^{j}}^{r}+\int_{0}^{r-n a^{j}}\right) t^{\alpha-1}\left\|T(r-t) f_{\delta}\right\|_{1} d t=I I I+I V,
$$

and

$$
\begin{aligned}
& \int_{r-n a^{j}}^{r} t^{\alpha-1}\left\|T(r-t) f_{\delta}\right\|_{1} d t \\
& \quad=\sum_{k=1}^{n}\left(\int_{r-k a^{j}}^{r-(k-1) a^{j}} t^{\alpha-1} d t \cdot \frac{\int_{r-k a^{j}}^{r-(k-1) a^{j}} t^{\alpha-1}\left\|T(r-t) f_{\delta}\right\|_{1} d t}{\int_{r-k a^{j}}^{r-(k-1) a^{j}} t^{\alpha-1} d t}\right) .
\end{aligned}
$$

We now estimate $I I I$ and $I V$. For the estimation of $I I I$ we first note: if $0 \leq p<q<w<\infty$, then for every $A>0$

$$
\frac{\int_{p}^{q} t^{\alpha-1} d t}{\int_{q}^{w} s^{\alpha-1} d s}>\frac{\int_{p+A}^{q+A} t^{\alpha-1} d t}{\int_{q+A}^{w+A} s^{\alpha-1} d s} .
$$

(In fact, if $0<\alpha<1$ and if $0 \leq p<t<q<s<w<\infty$, then for any $A>0$ we have

$$
(1+A / t)^{\alpha-1}<(1+A / q)^{\alpha-1}<(1+A / s)^{\alpha-1},
$$

and thus

$$
\begin{aligned}
\frac{\int_{p}^{q} t^{\alpha-1} d t}{\int_{q}^{w} s^{\alpha-1} d s} & =\frac{\int_{p}^{q} t^{\alpha-1}(1+A / q)^{\alpha-1} d t}{\int_{q}^{w} s^{\alpha-1}(1+A / q)^{\alpha-1} d s} \\
& >\frac{\int_{p}^{q} t^{\alpha-1}(1+A / t)^{\alpha-1} d t}{\int_{q}^{w} s^{\alpha-1}(1+A / s)^{\alpha-1} d s}=\frac{\int_{p+A}^{q+A} t^{\alpha-1} d t}{\int_{q+A}^{w+A} s^{\alpha-1} d s}
\end{aligned}
$$


which completes the proof of (4.12).)

Letting $s=t-\left(r-k a^{j}\right)$ for $r-k a^{j} \leq t<r-(k-1) a^{j}$, we have $0 \leq s<a^{j}$, and

$$
\begin{aligned}
& \left\|T(r-t) f_{\delta}\right\|_{1}=\left\|T\left(k a^{j}-s\right) f_{\delta}\right\|_{1}=\left\|T\left(a^{j}-s\right) f_{\delta}\right\|_{1} \\
& \leq \begin{cases}2^{j+1} & \left(0 \leq s<a^{0}=1 \text { or } r-k a^{j} \leq t<r-k a^{j}+1\right) \\
2^{j-l} & \left(a^{l} \leq s<a^{l+1} \text { or } r-k a^{j}+a^{l} \leq t<r-k a^{j}+a^{l+1}, 0 \leq l \leq j-1\right) .\end{cases}
\end{aligned}
$$

\section{Therefore}

$$
\begin{aligned}
& \frac{\int_{r-k a^{j}}^{r-(k-1) a^{j}} t^{\alpha-1}\left\|T(r-t) f_{\delta}\right\|_{1} d t}{\int_{r-k a^{j}}^{r-(k-1) a^{j}} t^{\alpha-1} d t} \\
& \leq \sum_{l=0}^{j-1}\left(\frac{\int_{r-k a^{j}+a^{l}}^{r-k a^{j}+a^{l+1}} t^{\alpha-1} d t}{\int_{r-k a^{j}}^{r-(k-1) a^{j}} t^{\alpha-1} d t}\right) 2^{j-l}+\left(\frac{\int_{r-k a^{j}}^{r-k a^{j}+1} t^{\alpha-1} d t}{\int_{r-k a^{j}}^{r-(k-1) a^{j}} t^{\alpha-1} d t}\right) 2^{j+1} .
\end{aligned}
$$

\section{Define}

$$
\begin{aligned}
\xi_{1} & :=\left(\int_{0}^{1} t^{\alpha-1} d t\right) /\left(\int_{0}^{a^{j}} t^{\alpha-1} d t\right), \\
\eta_{1} & :=\left(\int_{r-k a^{j}}^{r-k a^{j}+1} t^{\alpha-1} d t\right) /\left(\int_{r-k a^{j}}^{r-(k-1) a^{j}} t^{\alpha-1} d t\right), \\
\xi_{l+2} & :=\left(\int_{a^{l}}^{a^{l+1}} t^{\alpha-1} d t\right) /\left(\int_{0}^{a^{j}} t^{\alpha-1} d t\right),
\end{aligned}
$$

and

$$
\eta_{l+2}:=\left(\int_{r-k a^{j}+a^{l}}^{r-k a^{j}+a^{l+1}} t^{\alpha-1} d t\right) /\left(\int_{r-k a^{j}}^{r-(k-1) a^{j}} t^{\alpha-1} d t\right)
$$

for $0 \leq l \leq j-1$. Then it follows from (4.12) that $\xi_{l} / \xi_{l+1} \geq \eta_{l} / \eta_{l+1}$. Hence we can apply part (a) of Lemma 4.3 to infer that 


$$
\begin{aligned}
& \sum_{l=0}^{j-1}\left(\frac{\int_{r-k a^{j}+a^{l}}^{r-k a^{j}+a^{l+1}} t^{\alpha-1} d t}{\int_{r-k a^{j}}^{r-(k-1) a^{j}} t^{\alpha-1} d t}\right) 2^{j-l}+\left(\frac{\int_{r-k a^{j}}^{r-k a^{j}+1} t^{\alpha-1} d t}{\int_{r-k a^{j}}^{r-(k-1) a^{j}} t^{\alpha-1} d t}\right) 2^{j+1} \\
& \leq \sum_{l=0}^{j-1}\left(\frac{\int_{a^{l}}^{a^{l+1}} t^{\alpha-1} d t}{\int_{0}^{a^{j}} t^{\alpha-1} d t}\right)^{2^{j-l}}+\left(\frac{\int_{0}^{1} t^{\alpha-1} d t}{\int_{0}^{a^{j}} t^{\alpha-1} d t}\right)^{2^{j+1}}=\frac{2 D_{j}}{\int_{0}^{a^{j}} t^{\alpha-1} d t} \\
& =\frac{\alpha}{a^{j \alpha}} 2 D_{j}<2 \sum_{l=0}^{j}\left(\frac{2}{a^{\alpha}}\right)^{l} \quad \text { (by (4.4), (4.7)) }
\end{aligned}
$$

for each $1 \leq k \leq n$. Hence

$$
\begin{aligned}
I I I & <\frac{\alpha}{r^{\alpha}}\left(\sum_{k=1}^{n} \int_{r-k a^{j}}^{r-(k-1) a^{j}} t^{\alpha-1} d t\right) 2 \sum_{l=0}^{j}\left(\frac{2}{a^{\alpha}}\right)^{l} \\
& =\frac{\alpha}{r^{\alpha}}\left(\int_{r-n a^{j}}^{r} t^{\alpha-1} d t\right) 2 \sum_{l=0}^{j}\left(\frac{2}{a^{\alpha}}\right)^{l} .
\end{aligned}
$$

On the other hand we have

$$
\begin{aligned}
I V & =\frac{\alpha}{r^{\alpha}} \int_{0}^{r-n a^{j}} t^{\alpha-1}\left\|T\left(r-n a^{j}-t\right) f_{\delta}\right\|_{1} d t \\
& =\frac{\alpha}{r^{\alpha}}\left(\int_{0}^{r-n a^{j}} t^{\alpha-1} d t\right) \cdot\left\|C_{r-n a^{j}}^{\alpha} f_{\delta}\right\|_{1} \\
& <\frac{\alpha}{r^{\alpha}}\left(\int_{0}^{r-n a^{j}} t^{\alpha-1} d t\right) \cdot\left(4+8 \sum_{k=0}^{j-1}\left(\frac{2}{a^{\alpha}}\right)^{k}\right) \quad \text { (by (iii)). }
\end{aligned}
$$

Since $\frac{r^{\alpha}}{\alpha}=\int_{0}^{r} t^{\alpha-1} d t$, we deduce from these observations that

$$
\left\|C_{r}^{\alpha} f_{\delta}\right\|_{1}=I I I+I V<4+8 \sum_{k=0}^{j-1}\left(\frac{2}{a^{\alpha}}\right)^{k}+2 \sum_{k=0}^{j}\left(\frac{2}{a^{\alpha}}\right)^{k} \quad\left(r>a^{j}\right) .
$$

(v) Finally we estimate $\left\|C_{t}^{\alpha} g_{r}^{\prime}\right\|_{1}(t>0)$, where $g_{r}^{\prime}$ is the function defined in (4.10) for the case that $a^{j^{\prime}-1} \leq r-\delta^{\prime}<r<a^{j^{\prime}}$, with $1 \leq j^{\prime} \leq j$ and $0<\delta^{\prime}<1$. If $0<t \leq r$, then, as in (ii) and (iii), we have 


$$
\left\|C_{t}^{\alpha} g_{r}^{\prime}\right\|_{1}<\left(4+8 \sum_{k=0}^{j^{\prime}-1}\left(\frac{2}{a^{\alpha}}\right)^{k}\right) \leq\left(4+8 \sum_{k=0}^{j-1}\left(\frac{2}{a^{\alpha}}\right)^{k}\right) .
$$

And, if $t>r$, then we write

$$
\left\|C_{t}^{\alpha} g_{r}^{\prime}\right\|_{1}=\frac{\alpha}{t^{\alpha}}\left(\int_{t-r}^{t}+\int_{0}^{t-r}\right) s^{\alpha-1}\left\|T(t-s) g_{r}^{\prime}\right\|_{1} d s=V+V I,
$$

where

$$
\begin{aligned}
V & <\frac{\alpha}{t^{\alpha}} \int_{0}^{r} s^{\alpha-1}\left\|T(r-s) g_{r}^{\prime}\right\|_{1} d s<\left\|C_{r}^{\alpha} g_{r}^{\prime}\right\|_{1} \\
& <2+4 \sum_{k=0}^{j^{\prime}-1}\left(\frac{2}{a^{\alpha}}\right)^{k} \leq 2+4 \sum_{k=0}^{j-1}\left(\frac{2}{a^{\alpha}}\right)^{k} \quad \text { (cf. (4.8)). }
\end{aligned}
$$

On the other hand, since $T(r) g_{r}^{\prime}=\left(2^{\left(j-j^{\prime}\right)} \delta^{\prime}\right)^{-1} \chi_{\left[a^{j}-\delta^{\prime}, a^{j}\right)}=2^{-\left(j-j^{\prime}\right)} f_{\delta^{\prime}}$, it follows that

$$
\begin{aligned}
V I & =\frac{\alpha}{t^{\alpha}} \int_{0}^{t-r} s^{\alpha-1}\left\|T(t-r-s)\left(f_{\delta^{\prime}} / 2^{j-j^{\prime}}\right)\right\|_{1} d s \\
& <\frac{1}{2^{j-j^{\prime}}}\left\|C_{t-r}^{\alpha} f_{\delta^{\prime}}\right\|_{1} \leq\left\|C_{t-r}^{\alpha} f_{\delta^{\prime}}\right\|_{1} \\
& <4+8 \sum_{k=0}^{j-1}\left(\frac{2}{a^{\alpha}}\right)^{k}+2 \sum_{k=0}^{j}\left(\frac{2}{a^{\alpha}}\right)^{k},
\end{aligned}
$$

where the last inequality comes from (i), (iii) and (iv); in particular (iii) is applied to the function $g_{a^{j}}=f_{\delta^{\prime}}$.

Hence, if $t>r$, then

$$
\left\|C_{t}^{\alpha} g_{r}^{\prime}\right\|_{1}=V+V I<6+12 \sum_{k=0}^{j-1}\left(\frac{2}{a^{\alpha}}\right)^{k}+2 \sum_{k=0}^{j}\left(\frac{2}{a^{\alpha}}\right)^{k} .
$$

By this and (4.15),

$$
\sup _{0<\delta<1}\left\|C_{a^{j}}^{\alpha} f_{\delta}\right\|_{1} \leq \alpha\left(X_{j}\right)<6+14 \sum_{k=0}^{j}\left(\frac{2}{a^{\alpha}}\right)^{k}
$$

for each $j \geq 1$, so that we have:

(vii) $\gamma<\alpha<1$ implies $\sup _{j \geq 1} \alpha\left(X_{j}\right) \leq 6+14 a^{\alpha}\left(a^{\alpha}-2\right)^{-1}$ (since $a^{\alpha}>2$ );

(viii) $0<\alpha \leq \gamma$ implies $\lim _{j \rightarrow \infty} \alpha\left(X_{j}\right)=\infty$ (by (4.6)). 
Therefore, we conclude that

$$
\sup _{t>0}\left\|C_{t}^{\gamma}\right\|_{1}=\infty, \quad \text { and } \quad \sup _{t>0}\left\|C_{t}^{\beta}\right\|_{1}<\infty \quad \text { for all } \beta>\gamma .
$$

We are now in a position to consider the cosine operator function case. For $j \geq 1$, let

$$
Y_{j}:=\left[-a^{j}, a^{j}\right), \quad \text { and } \quad v_{j}(s):=w_{j}(|s|) \quad \text { for } s \in Y_{j} .
$$

For $t \in \mathbb{R}$, define a point transformation $\tau_{j, t}: Y_{j} \rightarrow Y_{j}$ by $\tau_{j, t}(s):=s+t(\bmod$ $2 a^{j}$ ), and put $S_{j}(t) f(s):=f\left(\tau_{j, t}(s)\right)$ for $f$ on $Y_{j}$. Define a measure $\nu_{j}$ on $Y_{j}$ by $\nu_{j}:=v_{j}(s) d s$.

Write

$$
Y:=\bigcup_{j=1}^{\infty} Y_{j} \quad \text { (regarded as a disjoint union), }
$$

and let $\nu$ be the measure on $Y$ defined by $\left.\nu\right|_{Y_{j}}:=\nu_{j}$ for each $j \geq 1$. For $t \in \mathbb{R}$, define an operator $S(t): L_{1}(Y, \nu) \rightarrow L_{1}(Y, \nu)$ by $\left.(S(t) f)\right|_{Y_{j}}:=S_{j}(t)\left(\left.f\right|_{Y_{j}}\right)$ for $j \geq 1$. It is clear that $S(\cdot)$ becomes a strongly continuous one-parameter group of positive linear operators on $L_{1}(Y, \nu)$. Thus, if we set $C(t):=2^{-1}(S(t)+S(-t))$ for $t \geq 0$, then $(C(t))_{t \geq 0}$ becomes a strongly continuous cosine operator function of positive linear operators on $L_{1}(Y, \nu)$. Then, by using part (b) of Lemma 4.3 and the above calculations, it is now routine to check that the Cesaro means $C_{t}^{\gamma}$ and $C_{t}^{\beta}$ of $(C(t))_{t \geq 0}$ satisfy (4.18).

This completes the proof of Theorem 4.2.

The above proof of Theorem 4.2 turns up the following result.

Theorem 4.4. There exists a $C_{0}$-semigroup $(T(t))_{t \geq 0}$ [resp. a strongly continuous cosine operator function $(C(t))_{t \geq 0}$ ] of positive linear operators on an $L_{1}$-space such that $\sup _{t>0}\|T(t)\|=\infty$ [resp. $\sup _{t>0}\|C(t)\|=\infty$ ], but $\sup _{t>0}\left\|C_{t}^{\gamma}\right\|<\infty$ for all $\gamma>0$ and, in particular, the Abel means of $(T(t))_{t \geq 0}\left[\right.$ resp. $\left.(C(t))_{t \geq 0}\right]$ are uniformly bounded.

Proof. We first note that if $a(\gamma)(>2)$ denotes the unique solution of the equation $2=a^{\gamma}$ for $0<\gamma<1$, then we have $a(\gamma) \uparrow \infty$ as $\gamma \downarrow 0$, and $\{a(\gamma): 0<\gamma<1\}=(2, \infty)$. Thus, for each $k \geq 1$, there exists a unique $\gamma_{k}$, with $0<\gamma_{k}<1$, such that $a\left(\gamma_{k}\right)=4^{k}$. Since $a(1 / k)=2^{k}<4^{k}=a\left(\gamma_{k}\right)$, it follows that $\gamma_{k}<1 / k$. By this, together with the proof of Theorem 4.2 (see especially (4.3), (4.17), and (vii), (viii) below (4.17)), we see that for each $k \geq 1$ there exists a $C_{0}$-semigroup $\left(T_{k}(t)\right)_{t \geq 0}$ of positive linear operators on $L_{1}\left(\Omega_{k}, \mu_{k}\right)$ such that 


$$
k<\sup _{t>0}\left\|T_{k}(t)\right\|_{1}<\infty, \quad \text { and } \quad \sup _{t>0}\left\|C_{t}^{1 / k}\left(T_{k}(\cdot)\right)\right\|_{1} \leq 34
$$

where the constant 34 comes from (vii) by putting $a=a\left(\gamma_{k}\right)=4^{k}$, with $\gamma=\gamma_{k}$ and $\alpha=1 / k$. Let

$$
\Omega:=\bigcup_{k=1}^{\infty} \Omega_{k} \quad \text { (regarded as a disjoint union), }
$$

and let $\mu$ be the measure on $\Omega$ defined by $\left.\mu\right|_{\Omega_{k}}:=\mu_{k}$ for each $k \geq 1$. For $t \geq 0$, define a positive linear operator $T(t): L_{1}(\Omega, \mu) \rightarrow L_{1}(\Omega, \mu)$ by

$$
\left.(T(t) f)\right|_{\Omega_{k}}:=T_{k}(t)\left(\left.f\right|_{\Omega_{k}}\right) \quad(k \geq 1) .
$$

It follows that if $0 \leq f \in L_{1}(\Omega, \mu)$ then $0 \leq T(t) f \in L_{1}(\Omega, \mu)$, and $\lim _{t \downarrow} \| T(t) f-$ $f \|_{1}=0$. Thus $(T(t))_{t \geq 0}$ is a $C_{0}$-semigrou $\mathrm{p}$ on $L_{1}(\Omega, \mu)$, and we have $\sup _{t>0}\|T(t)\|_{1}=\infty$, because $\sup _{t>0}\|T(t)\|_{1} \geq \sup _{t>0}\left\|T_{k}(t)\right\|_{1}>k$ for $k \geq 1$ by (4.19). On the other hand, if $\gamma>0$, then, choose $n \geq 1$ so that $1 / n<\gamma$. Then we have by Theorem 2.2(i)

$$
\begin{aligned}
\sup _{t>0}\left\|C_{t}^{\gamma}\right\|_{1} & \leq \sup _{t>0}\left\|C_{t}^{1 / n}\right\|_{1} \\
& \leq \max _{1 \leq k<n}\left(\sup _{t>0}\left\|T_{k}(t)\right\|_{1}\right)+\sup _{k \geq n}\left(\sup _{t>0}\left\|C_{t}^{1 / n}\left(T_{k}(\cdot)\right)\right\|_{1}\right) \\
& \left.\leq \max _{1 \leq k<n}\left(\sup _{t>0}\left\|T_{k}(t)\right\|_{1}\right)+\sup _{k \geq n} \sup _{t>0}\left\|C_{t}^{1 / k}\left(T_{k}(\cdot)\right)\right\|_{1}\right) \\
& \left.\leq \max _{1 \leq k<n}\left(\sup _{t>0}\left\|T_{k}(t)\right\|_{1}\right)+34<\infty \quad \text { (by }(4.19)\right) .
\end{aligned}
$$

This completes the proof for the semigroup case.

The proof for the cosine operator function case is similar, and hence we may omit the details.

\section{REFERENCES}

1. W. Arendt, C. J. K. Batty, M. Hieber and F. Neubrander, Vector-valued Laplace Transforms and Cauchy Problems, Monographs in Mathematics Vol. 96, Birkhauser Verlag, 2001.

2. E. Artin, Einfuhrung in die Theorie der Gammafunktion, Hamburg, 1931.

3. T. Eisner and H. Zwart, Continuous-time Kreiss resolvent condition on infinitedimensional spaces, Math. Comp. 75 (2006), 1971-1985. 
4. T. Eisner and H. Zwart, A note on polynomially growing $C_{0}$-semigroups, Semigroup Forum 75 (2007), 438-445.

5. A. M. Gomilko, On conditions for the generating operator of a uniformly bounded $C_{0}$-semigroup of operators, Funct. Anal. Appl. 33 (1999), 294-296.

6. E. Hille and R. S. Phillips, Functional Analysis and Semigroups, Amer. Math. Soc. Colloq. Publ., Vol. 31, Amer. Math. Soc., Providence, R. I., 1957.

7. Y.-C. Li, R. Sato and S.-Y. Shaw, Convergence theorems and Tauberian theorems for functions and sequences in Banach spaces and Banach lattices, Israel J. Math. 162 (2007), 109-149.

8. Y.-C. Li, R. Sato and S.-Y. Shaw, Boundedness and growth orders of means of discrete and continuous semigroups of operators, Studia Math. 187 (2008), 1-35.

9. M. Malejki, $C_{0}$-groups with polynomial growth, Semigroup Forum 67 (2003), 63-75.

10. R. Sato, On ergodic averages and the range of a closed operator, Taiwanese J. Math., 10 (2006), 1193-1223.

11. R. Sato and S.-Y. Shaw, Strong and uniform mean stability of cosine and sine operator functions, J. Math. Anal. Appl. 330 (2007), 1293-1306.

12. M. Sova, Cosine operator functions, Rozprawy Math. 49 (1966), 1-47.

13. T. Takagi, Kaisekigairon, 3rd edition, Iwanami, Tokyo, 1967, (in Japanese).

Jeng-Chung Chen

Department of Applied Mathematics,

National Hsin-Chu University of Education,

Hsin-Chu 300,

Taiwan

E-mail: jcchen@mail.nhcue.edu.tw

Ryotaro Sato

Department of Mathematics,

Okayama University,

Okayama 700-8530,

Japan

Current address:

19-18, Higashi-hongo 2-chome,

Midori-ku, Yokohama 226-0002,

Japan

E-mail: satoryot@math.okayama-u.ac.jp

Sen-Yen Shaw

Graduate School of Engineering,

Lunghwa University of Science and Technology,

Gueishan, Taoyuan 333,

Taiwan

E-mail: shaw @ math.ncu.edu.tw 